\title{
The local Donaldson-Thomas theory of curves
}

\author{
A OKOUNKOV \\ R PANDHARIPANDE
}

\begin{abstract}
The local Donaldson-Thomas theory of curves is solved by localization and degeneration methods. The results complete a triangle of equivalences relating Gromov-Witten theory, Donaldson-Thomas theory, and the quantum cohomology of the Hilbert scheme of points of the plane.
\end{abstract}

$14 \mathrm{~N} 10$

Dedicated to the memory of Raoul Bott

\section{Introduction}

\subsection{Overview}

Let $X$ be a nonsingular projective variety of dimension 3 over $\mathbb{C}$. Gromov-Witten theory is defined by integration over the moduli space of stable maps to $X$, and Donaldson-Thomas theory is defined by integration over the moduli space of ideal sheaves of $X$ (see Donaldson-Thomas [5], Maulik et al [24; 25] and Thomas [34]).

If $X$ is quasi-projective, the Gromov-Witten and Donaldson-Thomas theories may not be well-defined. However, if $X$ is the total space of a rank 2 bundle over a nonsingular projective curve,

$$
N \rightarrow C,
$$

local Gromov-Witten and Donaldson-Thomas theories are defined via equivariant residues (see Bryan-Pandharipande [4] and Maulik et al [24]).

The Gromov-Witten and Donaldson-Thomas theories of $X$ relative to a nonsingular surface

$$
S \subset X
$$

are defined via moduli spaces of maps and sheaves with boundary conditions along $S$. See Eliashberg-Givental-Hofer [6], Ionel-Parker [12], Li-Ruan [16], Li [17; 18] and Maulik et al [25] for various treatments of the subject. 
If $X$ is the total space of a rank 2 bundle, a natural set of surfaces is determined by the fibers of $N$ over points of $C$. The data

$$
\left(N \rightarrow C, p_{1}, \ldots, p_{r}\right)
$$

determine relative local Gromov-Witten and Donaldson-Thomas theories of $N$ over $C$.

The local theory of curves refers to all relative local Gromov-Witten and DonaldsonThomas theories ${ }^{1}$ specified by data of type (1). The main result of the paper is a proof of the Gromov-Witten/Donaldson-Thomas correspondence for the local theory of curves.

The local Gromov-Witten theory of curves is determined in Bryan-Pandharipande [4]. Our focus here is on the local Donaldson-Thomas theory of curves. The paper has at least three motivations:

(i) local curves provide a rich class of non-toric examples of the GW/DT correspondence,

(ii) the proof yields a computation of the 1-legged equivariant vertex,

(iii) the correspondence for local curves will likely play a basic role in the proof of the GW/DT correspondence for all 3-folds.

The 1-legged equivariant vertex (ii) contains the Calabi-Yau 1-legged vertex governed by the Gopakumar-Mariño-Vafa formula (see Liu-Liu-Zhou [21], Mariño-Vafa [23] and Okounkov-Pandharipande [29]) as a special case. The computation of the 1-legged equivariant vertex may be viewed as a Hodge integral result on the Gromov-Witten side or a vertex measure result on the Donaldson-Thomas side.

The main foundational reference for Donaldson-Thomas theory in algebraic geometry is Thomas [34], see also Maulik et al [24]. The required foundational development of relative Donaldson-Thomas theory has yet to be written. ${ }^{2}$ We will assume two basic properties of relative Donaldson-Thomas theory: existence and degeneration (see Maulik et al [25]).

\subsection{Definitions}

1.2.1 Ideal sheaves Let $C$ be a nonsingular projective curve, and let $N$ be the total space of a rank 2 bundle,

$$
N \rightarrow C \text {. }
$$

\footnotetext{
${ }^{1}$ We study here theories with no internal insertions.

${ }^{2} \mathrm{See} \mathrm{Li}-\mathrm{Wu}[19]$ for recent developments.
} 
Let $I_{n}(N, d)$ denote the moduli space of of ideal sheaves

$$
0 \longrightarrow I_{Z} \longrightarrow \mathcal{O}_{N} \longrightarrow \mathcal{O}_{Z} \longrightarrow 0
$$

of proper subschemes $Z \subset N$ of degree $d$ and Euler characteristic

$$
n=\chi\left(\mathcal{O}_{Z}\right) \text {. }
$$

The degree of $Z$ is simply the length of the intersection

$$
Z \cap N_{p}
$$

where $p \in C$ is a generic point.

1.2.2 Partition functions If $N$ decomposes as a direct sum of line bundles,

$$
N=L_{1} \oplus L_{2}
$$

the splitting determines a scaling action of a 2-dimensional torus $T$ on $N$. The level of the splitting is the pair of integers $\left(k_{1}, k_{2}\right)$ where,

$$
k_{i}=\operatorname{deg}\left(L_{i}\right) .
$$

Of course, the scaling action and the level depend upon the splitting (2).

Since every $T$-fixed proper subscheme $Z$ must be supported on the zero section of $N$, the $T$-fixed point set

$$
I_{n}(N, d)^{T} \subset I_{n}(N, d)
$$

is proper.

The moduli space $I_{n}(N, d)$ carries a $T$-equivariant perfect obstruction theory obtained from (traceless) $\operatorname{Ext}_{0}(I, I)$, see Thomas [34]. Though $N$ is quasi-projective, $\operatorname{Ext}_{0}(I, I)$ is well-defined since the associated quotient scheme $Z \subset N$ is proper. Alternatively, for any $T$-equivariant compactification,

$$
N \subset \bar{N}
$$

the obstruction theory on

$$
I_{n}(N, d) \subset I_{n}(\bar{N}, d)
$$

is obtained by restriction.

The definition of the local Donaldson-Thomas invariants of $N$ follows the strategy of Bryan-Pandharipande [4] and Maulik et al [24]. We define $\mathrm{Z}(N)_{d}$ formally by:

$$
\mathrm{Z}(N)_{d} "=" \sum_{n \in \mathbb{Z}} q^{n} \int_{\left[I_{n}(N, d)\right]^{\mathrm{vir}}} 1 .
$$


The variable $q$ indexes the Euler characteristic $n$. The integral on the right of (3) is well-defined by the virtual localization formula as an equivariant residue.

Definition 1 The partition function for the degree $d$ local Donaldson-Thomas invariants of $N$ is defined by:

$$
\mathrm{Z}(N)_{d}=\sum_{n \in \mathbb{Z}} q^{n} \int_{\left[I_{n}(N, d)^{T}\right]^{\mathrm{vir}}} \frac{1}{e\left(\mathrm{Norm}^{\mathrm{vir}}\right)} .
$$

The $T$-fixed part of the perfect obstruction theory for $I_{n}(N, d)$ induces a perfect obstruction theory for $I_{n}(N, d)^{T}$ and hence a virtual class (see Graber-Pandharipande [9] and Maulik et al [24]). The equivariant virtual normal bundle of the embedding,

$$
I_{n}(N, d)^{T} \subset I_{n}(N, d),
$$

is Norm ${ }^{\text {vir }}$ with equivariant Euler class $e\left(\right.$ Norm $\left.^{\text {vir }}\right)$. The integral in (4) denotes equivariant push-forward.

Following the notation of Maulik et al [24;25], Z(N) $d$ is unprimed since the degree 0 contributions have not yet been removed. Since a geometrical method of removing the degree 0 contribution from Donaldson-Thomas theory is not available, a formal method is followed.

Definition 2 The reduced partition function $\mathrm{Z}_{D T}^{\prime}(N)_{d}$ for the degree $d$ local Donaldson-Thomas invariants of $N$ is defined by:

$$
\mathrm{Z}^{\prime}(N)_{d}=\frac{\mathrm{Z}(N)_{d}}{\mathrm{Z}(N)_{0}}
$$

The residues defined by the localization formula take values in the localized $T$ equivariant cohomology of a point,

$$
\int_{\left[I_{n}(N, d)^{T}\right]^{\mathrm{vir}}} \frac{1}{e\left(\mathrm{Norm}^{\mathrm{vir}}\right)} \in \mathbb{Q}\left(t_{1}, t_{2}\right) .
$$

Here, $t_{1}, t_{2}$ are the weights of the standard representations of the factors of $T$.

If $N$ is an indecomposable rank 2 vector bundle, the total space of $N$ admits a scaling action of a 1-dimensional algebraic torus. The local Donaldson-Thomas theory of $N$ can be defined as above with respect to the 1-dimensional scaling torus. However, since every indecomposable bundle $N$ is deformation equivalent to a split bundle over $C$, the indecomposable case is recovered from the split case via restriction to the diagonal torus.

In our study of the local Donaldson-Thomas theory of $N$, we will always assume a splitting (2) of $N$ and an action of a 2-dimensional scaling torus $T$. 
1.2.3 Relative geometry The fiber of $N$ over a point $p \in C$ determines a $T-$ equivariant divisor

$$
N_{p} \subset N
$$

isomorphic to $\mathbb{C}^{2}$ with the standard $T$-action. We will consider the local theories of $N$ relative to the divisor

$$
S=\bigcup_{i=1}^{r} N_{p_{i}} \subset N
$$

determined by the fibers over $p_{1}, \ldots, p_{r} \in C$.

Let $I_{n}(N / S, d)$ denote the relative moduli space of ideal sheaves, see Li [18] and Maulik et al [25] for a discussion. The construction of $I_{n}(N / S, d)$, and the existence of a canonical $T$-equivariant $\operatorname{Ext}_{0}(I, I)$ obstruction theory will be assumed here.

For each $p_{i}$, let $\eta^{i}$ be a partition of $d$ weighted by the equivariant Chow ring,

$$
A_{T}^{*}\left(N_{p_{i}}, \mathbb{Q}\right) \cong \mathbb{Q}\left[t_{1}, t_{2}\right],
$$

of the fiber $N_{p_{i}}$. By Nakajima's construction, a weighted partition $\eta^{i}$ determines a $T$-equivariant class

$$
C_{\eta^{i}} \in A_{T}^{*}\left(\operatorname{Hilb}\left(N_{p_{i}}, d\right), \mathbb{Q}\right)
$$

in the Chow ring of the Hilbert scheme of points. In Donaldson-Thomas theory, the weighted partition $\eta^{i}$ specifies relative conditions via the boundary map

$$
\epsilon_{i}: I_{n}(N / S, d) \rightarrow \operatorname{Hilb}\left(N_{p_{i}}, d\right) .
$$

An element $\eta \in \mathcal{P}(d)$ of the set of partitions of $d$ may be viewed as a weighted partition with all weights set to the identity class $1 \in H_{T}^{*}\left(N_{p_{i}}, \mathbb{Q}\right)$. The Nakajima basis of $A_{T}^{*}\left(\operatorname{Hilb}\left(N_{p_{i}}, d\right), \mathbb{Q}\right)$ consists of identity weighted partitions indexed by $\mathcal{P}(d)$. The $T$-equivariant intersection pairing in the Nakajima basis is:

$$
\int_{\operatorname{Hilb}\left(N_{p_{i}}, d\right)} C_{\mu} \cup C_{v}=\frac{1}{\left(t_{1} t_{2}\right)^{\ell(\mu)}} \frac{(-1)^{d-\ell(\mu)}}{\mathfrak{z}(\mu)} \delta_{\mu, \nu},
$$

where

$$
\mathfrak{z}(\mu)=\prod_{i=1}^{\ell(\mu)} \mu_{i} \cdot|\operatorname{Aut}(\mu)| .
$$

The notation $\eta([0])$ will be used to set all weights to $[0] \in A_{T}^{*}\left(N_{p_{i}}, \mathbb{Q}\right)$. Since

$$
[0]=t_{1} t_{2} \in A_{T}^{*}\left(N_{p_{i}}, \mathbb{Q}\right),
$$

the weight choice has only a mild effect. 
Following the notation of Bryan-Pandharipande [4] and Maulik et al [25], the relative local Donaldson-Thomas partition function,

$$
\mathrm{Z}(N / S)_{d, \eta^{1}, \ldots, \eta^{r}}=\sum_{n \in \mathbb{Z}} q^{n} \int_{\left[I_{n}(N / S, d)^{T}\right]^{\mathrm{vir}}} \frac{\prod_{i=1}^{r} \epsilon_{i}^{*}\left(C_{\eta^{i}}\right)}{e\left(\mathrm{Norm}^{\mathrm{vir}}\right)},
$$

is well-defined. Let

$$
\mathrm{Z}^{\prime}(N / S)_{d, \eta^{1}, \ldots, \eta^{r}}=\frac{\mathrm{Z}(N / S)_{d, \eta^{1}, \ldots, \eta^{r}}}{\mathrm{Z}(N / S)_{0}} .
$$

denote the reduced relative partition function.

\subsection{Degeneration}

Simpler notation for the partition functions will often be used. If $N$ is split with level $\left(k_{1}, k_{2}\right)$ over a genus $g$ base curve $C$, then let

$$
\begin{gathered}
\mathrm{Z}\left(g \mid k_{1}, k_{2}\right)_{d, \eta^{1}, \ldots, \eta^{r}}=\mathrm{Z}(N / S)_{d, \eta^{1}, \ldots, \eta^{r}}, \\
\mathrm{Z}^{\prime}\left(g \mid k_{1}, k_{2}\right)_{d, \eta^{1}, \ldots, \eta^{r}}=\mathrm{Z}^{\prime}(N / S)_{d, \eta^{1}, \ldots, \eta^{r}} .
\end{gathered}
$$

For many formulas, the $q$-shifted function,

$$
\mathrm{DT}\left(g \mid k_{1}, k_{2}\right)_{d, \eta^{1}, \ldots, \eta^{r}}=q^{-d(1-g)} \mathrm{Z}^{\prime}\left(g \mid k_{1}, k_{2}\right)_{d, \eta^{1}, \ldots, \eta^{r}},
$$

will be more convenient. In the relative cases, the redundant degree subscript $d$ will often be dropped.

Let $\Delta_{d}$ be the inverse of the $T$-equivariant intersection form of the Nakajima basis of $\operatorname{Hilb}\left(\mathbb{C}^{2}, d\right)$,

$$
\triangle_{d}(\mu, v)=(-1)^{d-\ell(\mu)}\left(t_{1} t_{2}\right)^{\ell(\mu)} \mathfrak{z}(\mu) \delta_{\mu, \nu}
$$

The indices of the DT partition function are raised by $\Delta_{d}$ :

$$
\mathrm{DT}\left(g \mid k_{1}, k_{2}\right)_{\mu^{1}, \ldots, \mu^{s}}^{v^{1}, \ldots, v^{t}}=\mathrm{DT}\left(g \mid k_{1}, k_{2}\right)_{\mu^{1}, \ldots, \mu^{s}, v^{1}, \ldots, v^{t}} \prod_{i=1}^{t} \triangle_{d}\left(v^{i}, v^{i}\right) .
$$

All the partition functions discussed here lie in the ring $\mathbb{Q}\left(t_{1}, t_{2}\right)((q))$ of Laurent series in $q$.

The degeneration formulas for the local Donaldson-Thomas theory of curves are conveniently expressed in terms of the DT partition functions:

$$
\operatorname{DT}\left(g \mid k_{1}, k_{2}\right)_{\mu^{1}, \ldots, \mu^{s}}^{v^{1}, \ldots, v^{t}}=\sum_{\gamma} \mathrm{DT}\left(g^{\prime} \mid k_{1}^{\prime}, k_{2}^{\prime}\right)_{\mu^{1}, \ldots, \mu^{s}}^{\gamma} \mathrm{DT}\left(g^{\prime \prime} \mid k_{1}^{\prime \prime}, k_{2}^{\prime \prime}\right)_{\gamma}^{v^{1}, \ldots, v^{t}},
$$


where $g=g^{\prime}+g^{\prime \prime}$, and $k_{i}=k_{i}^{\prime}+k_{i}^{\prime \prime}$, and

$$
\mathrm{DT}\left(g \mid k_{1}, k_{2}\right)_{\mu^{1}, \ldots, \mu^{s}}=\sum_{\gamma} \mathrm{DT}\left(g-1 \mid k_{1}, k_{2}\right)_{\mu^{1}, \ldots, \mu^{s}, \gamma}^{\gamma},
$$

see Maulik et al [25] for a discussion. The above degeneration formulas will be assumed here.

\subsection{Localization}

Our localization formulas trace their origins to Bott's remarkable paper [1] with many stops along the way.

The virtual localization formula of Graber-Pandharipande [9], proven in the abstract setting of perfect obstruction theories, applies to the Donaldson-Thomas virtual class. Applications in absolute Donaldson-Thomas theory (without relative conditions) are treated foundationally in Maulik et al [24]. Applications in the relative setting follow from the existence of a $T$-equivariant $\operatorname{Ext}_{0}(I, I)$ obstruction theory.

\subsection{GW/DT correspondence}

Let $N$ be the a rank 2 bundle over a genus $g$ curve $C$ with splitting of level $\left(k_{1}, k_{2}\right)$. The GW/DT correspondence for the local theory of curves consists of three results.

First, the relative local degree 0 series of $N$ is determined in terms of the McMahon function,

$$
M(q)=\prod_{n \geq 1} \frac{1}{\left(1-q^{n}\right)^{n}},
$$

the generating series for 3-dimensional partitions.

Theorem 1 The degree 0 Donaldson-Thomas partition function is determined by:

$$
\mathrm{Z}(N / S)_{0}=M(-q)^{\int_{N} c_{3}\left(T_{N}[-S] \otimes K_{N}[S]\right)} .
$$

Here, $T_{N}[-S]$ is the sheaf of tangent fields on $N$ with logarithmic zeros, $K_{N}[S]$ is the logarithmic canonical bundle, and the integral in the exponent is defined via localization on $N$,

$$
\begin{aligned}
\int_{N} c_{3}\left(T_{N}[-S] \otimes K_{N}[S]\right) & =\int_{C} \frac{c_{3}\left(T_{N}[-S] \otimes K_{N}[S]\right)}{e(N)} \\
& =(2 g-2+r) \frac{\left(t_{1}+t_{2}\right)^{2}}{t_{1} t_{2}}-\left(k_{1}+k_{2}\right),
\end{aligned}
$$


where $r$ is the number of relative points on $C$.

Second, the reduced Donaldson-Thomas series are proven to satisfy a basic rationality condition.

Theorem 2 The reduced series $Z^{\prime}(N / S)_{d, \eta^{1}, \ldots, \eta^{r}}$ is a rational function in the variables $t_{1}, t_{2}$, and $q$.

Finally, the local Gromov-Witten theory and the local Donaldson-Thomas theories of curves are proven to exactly match.

Theorem 3 After the change of variables $e^{i u}=-q$,

$$
\begin{aligned}
&(-i u)^{d\left(2-2 g+k_{1}+k_{2}\right)-\delta} \mathrm{Z}_{G W}^{\prime}(N / S)_{d, \eta^{1}, \ldots, \eta^{r}}= \\
&(-q)^{-\frac{d}{2}\left(2-2 g+k_{1}+k_{2}\right)} \mathrm{Z}_{D T}^{\prime}(N / S)_{d, \eta^{1}, \ldots, \eta^{r}},
\end{aligned}
$$

For the Gromov-Witten side, we follow the definitions and notations of Bryan-Pandharipande [4]. In particular,

$$
\delta=\sum_{i=1}^{r} d-\ell\left(\eta^{i}\right)
$$

\subsection{Method}

Theorems 1-3 are proven by solving the local Donaldson-Thomas theory of curves. The GW/DT correspondence is obtained by matching the Gromov-Witten results of [4] with the Donaldson-Thomas results here.

The solution of the local Donaldson-Thomas theory of curves follows the TQFT strategy of [4]. The first step is the determination of the level $(0,0)$ theory of $\mathbf{P}^{1}$. In the Gromov-Witten case, integral evaluations over the moduli space of curves are required [4]. Parallel Donaldson-Thomas integrals are evaluated here via connections to the quantum cohomology of the Hilbert scheme points of the plane. Next, the level $(-1,0)$ theory of $\mathbf{P}^{1}$ is determined by a direct calculation. Together, the results solve the local Donaldson-Thomas theory of curves and prove the GW/DT correspondence.

The GW/DT correspondence for the local theory of curves has been studied in the absolute case for the anti-diagonal action by Y Song. The correspondence for $\mathbf{P}^{2}-$ bundles over curves is closely related (see Gholampour [7] and Gholampour-Song [8]). 


\subsection{Quantum cohomology of $\operatorname{Hilb}\left(\mathbb{C}^{2}, d\right)$}

For $\lambda, \mu, v \in \mathcal{P}(d)$, define the series $\langle\lambda, \mu, v\rangle^{\text {Hilb }_{d}}$ of genus 0,3 -pointed, $T$-equivariant Gromov-Witten invariants of $\operatorname{Hilb}\left(\mathbb{C}^{2}, d\right)$ by a sum over curve degrees

$$
\langle\lambda, \mu, v\rangle^{\mathrm{Hilb}_{d}}=\sum_{n \geq 0} q^{n}\langle\lambda, \mu, v\rangle_{0,3, n}^{\mathrm{Hilb}_{d}} .
$$

The insertions on the right stand for Nakajima basis elements. See Okounkov-Pandharipande [31] for a complete discussion of the Gromov-Witten invariants of the Hilbert scheme $\operatorname{Hilb}\left(\mathbb{C}^{2}, d\right)$.

The results of [31] together with our calculation of the local Donaldson-Thomas theory of curves yields a Donaldson-Thomas/Hilbert correspondence.

Theorem $4 \operatorname{DT}(0 \mid 0,0)_{d, \lambda, \mu, v}=\langle\lambda, \mu, v\rangle^{\mathrm{Hilb}_{d}}$.

Our results complete the triangle of equivalences studied in the earlier papers:

Quantum cohomology of $\mathrm{Hilb}_{d}\left(\mathbb{C}^{2}\right)[31]$

Gromov-Witten theory of $\mathbf{P}^{1} \times \mathbb{C}^{2}$ [4] A fourth vertex of equivalence is obtained from the orbifold quantum cohomology of the stack symmetric product $\left(\mathbb{C}^{2}\right)^{d} / \Sigma_{d}$, see Bryan-Graber [2].

\subsection{Acknowledgments}

We thank J Bryan, G Farkas, T Graber, J Li, D Maulik, and N Nekrasov for many valuable discussions. We thank $\mathbf{J}$ Kock for the use of his cobordism LTEX macros.

Parts of the paper were written during a workshop on algebraic geometry and topological strings at the Instituto Superior Técnico in Lisbon in the fall of 2005. Both authors were partially supported by the Packard foundation and the NSF.

\section{Degree 0}

\subsection{Vertex measures}

The degree 0 calculation of the local Donaldson-Thomas theory of curves is easily obtained from the results of Maulik et al [24; 25]. Let

$$
\mathrm{W}(\varnothing, \varnothing, \varnothing)=M(-q)^{-\frac{\left(s_{1}+s_{2}\right)\left(s_{1}+s_{3}\right)\left(s_{2}+s_{3}\right)}{s_{1} s_{2} s_{3}}}
$$


denote the generating series of the equivariant vertex measures of finite 3-dimensional partitions at a 3-fold fixed point with tangent weights $s_{1}, s_{2}, s_{3}$. Let

$$
\mathrm{W}(\varnothing, \varnothing, \varnothing)_{S}=M(-q)^{-\frac{s_{2}+s_{3}}{s_{1}}}
$$

denote series of the finite vertex measures at a 3 -fold fixed point on a relative divisor $S$ with normal weight $s_{1}$ and tangent weights $s_{2}, s_{3}$.

The evaluations (6) and (7) are Theorem 1 and Corollary 1 of [25] with an appropriate adjustment of signs to match the conventions here.

\subsection{Localization}

Let $S$ denote the 1-dimension torus acting on $\mathbf{P}^{1}$ with respective tangent weights $S$ and $-s$ at the fixed points $0, \infty \in \mathbf{P}^{1}$.

Let $N=L_{1} \oplus L_{2}$ be a splitting of level $\left(k_{1}, k_{2}\right)$ over $\mathbf{P}^{1}$. The $S$-action on $\mathbf{P}^{1}$ can be lifted to $N$ with fiber weights $\left(k_{1} s, k_{2} s\right)$ over $0 \in \mathbf{P}^{1}$ and fiber weights $(0,0)$ over $\infty \in \mathbf{P}^{1}$. Since the scaling $T$-action on $N$ commutes with $S$, the 3 -dimensional torus,

$$
\mathbf{T}=S \times T
$$

acts on $N$.

We may calculate the degree 0 Donaldson-Thomas series $\mathrm{Z}\left(0 \mid k_{1}, k_{2}\right)_{0}$ via virtual localization with respect to $\mathbf{T}$, see Graber-Pandharipande [9] and Maulik et al [24; 25]. By the evaluation of the finite vertex measure (6),

$$
\begin{aligned}
\mathrm{Z}\left(0 \mid k_{1}, k_{2}\right)_{0} & =\left[\left.\left.\mathrm{W}(\varnothing, \varnothing, \varnothing)\right|_{s, t_{1}+k_{1} s, t_{2}+k_{2} s} \cdot \mathrm{W}(\varnothing, \varnothing, \varnothing)\right|_{-s, t_{1}, t_{2}}\right]_{s=0} \\
& =M(-q)^{-2 \frac{\left(t_{1}+t_{2}\right)^{2}}{t_{1} t_{2}}-\left(k_{1}+k_{2}\right)} .
\end{aligned}
$$

The series $\mathrm{Z}\left(0 \mid k_{1}, k_{2}\right)_{0, \varnothing}$ relative to $\infty \in \mathbf{P}^{1}$ is also determined by virtual localization. Here, measure (6) arises at the $\mathbf{T}$-fixed point over 0 , and measure (7) arises at the $\mathbf{T}$-fixed point over $\infty$ of the relative divisor:

$$
\begin{aligned}
\mathrm{Z}\left(0 \mid k_{1}, k_{2}\right)_{0, \varnothing} & =\left[\left.\left.\mathrm{W}(\varnothing, \varnothing, \varnothing)\right|_{s, t_{1}+k_{1} s, t_{2}+k_{2} s} \cdot \mathrm{W}(\varnothing, \varnothing, \varnothing)_{N_{\infty}}\right|_{-s, t_{1}, t_{2}}\right]_{s=0} \\
& =M(-q)^{-\frac{\left(t_{1}+t_{2}\right)^{2}}{t_{1} t_{2}}-\left(k_{1}+k_{2}\right)} .
\end{aligned}
$$

By the above evaluations, the proof of Theorem 1 is complete for the cases $Z\left(0 \mid k_{1}, k_{2}\right)_{0}$ and $\mathrm{Z}\left(0 \mid k_{1}, k_{2}\right)_{0, \varnothing}$. 


\subsection{Degeneration}

The degeneration formulas for the degree 0 theory take the following two forms:

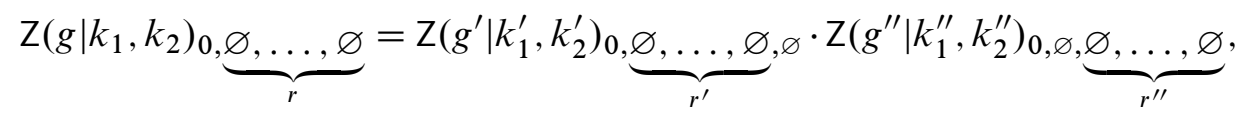

where $g=g^{\prime}+g^{\prime \prime}, k_{i}=k_{i}^{\prime}+k_{i}^{\prime \prime}, r=r^{\prime}+r^{\prime \prime}$, and

$$
\mathrm{Z}\left(g \mid k_{1}, k_{2}\right)_{0}, \underbrace{\varnothing, \ldots, \varnothing}_{r}=\mathrm{Z}\left(g-1 \mid k_{1}, k_{2}\right)_{0,} \underbrace{\varnothing, \ldots, \varnothing}_{r}, \varnothing, \varnothing .
$$

The first degeneration formula yields a recursive equation for $r \geq 2$ :

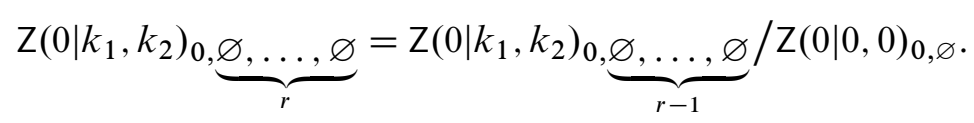

From the $r=0,1$ cases, we deduce

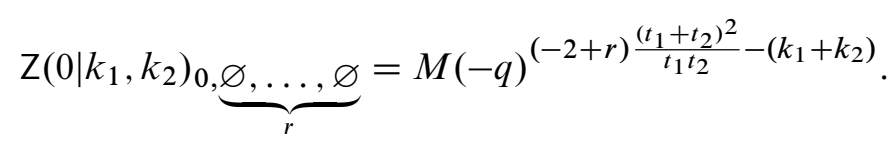

Finally, Theorem 1 is obtained for $g>0$ by applications of the second degeneration formula.

\section{TQFT}

\subsection{Overview}

The degeneration structure of local Donaldson-Thomas theory of curves is most concisely formulated as a functor of tensor categories,

$$
\text { DT(-): } 2 \mathbf{C o b}^{L_{1}, L_{2}} \rightarrow R \bmod .
$$

Our treatment here exactly follows the TQFT construction in Bryan-Pandharipande [4] for the local Gromov-Witten theory of curves. A more detailed discussion can be found there.

\section{2 $2 \operatorname{Cob}^{L_{1}, L_{2}}$}

The objects of the category $2 \mathbf{C o b}^{L_{1}, L_{2}}$ are compact oriented 1-manifolds. A morphism in $2 \mathbf{C o b}^{L_{1}, L_{2}}$,

$$
Y_{1} \rightarrow Y_{2}
$$


is an equivalence class of triples $\left(W, L_{1}, L_{2}\right)$ where $W$ is an oriented cobordism from $Y_{1}$ to $Y_{2}$ and $L_{1}, L_{2}$ are complex line bundles on $W$, trivialized on $\partial W$. The triples $\left(W, L_{1}, L_{2}\right)$ and $\left(W^{\prime}, L_{1}^{\prime}, L_{2}^{\prime}\right)$ are equivalent if there exists a boundary preserving oriented diffeomorphism,

$$
f: W \rightarrow W^{\prime}
$$

and bundle isomorphisms

$$
L_{i} \cong f^{*} L_{i}^{\prime}
$$

Composition is given by concatenation of the cobordisms and gluing of the bundles along the concatenation using the trivializations.

The isomorphism class of $L_{i}$ is determined by the Euler class

$$
e\left(L_{i}\right) \in H^{2}(W, \partial W)
$$

which assigns an integer to each component of $W$. For a connected cobordism $W$, we refer to the pair of integers $\left(k_{1}, k_{2}\right)$, determined by the Euler classes of $L_{1}$ and $L_{2}$, as the level. Under concatenation, the levels simply add. For example:
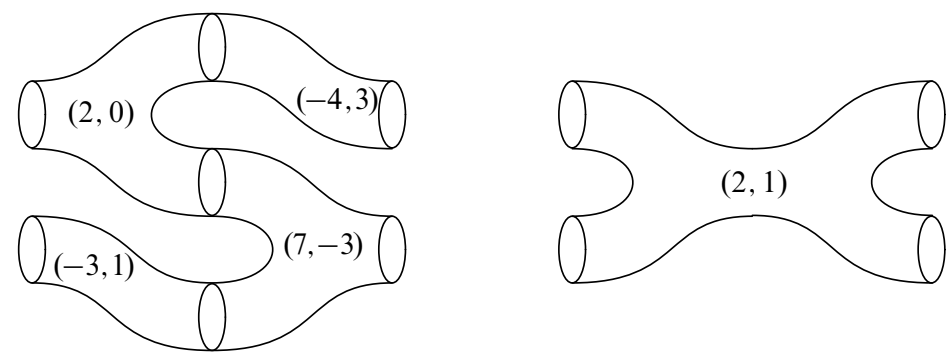

The empty manifold is a distinguished object in $2 \mathbf{C o b}^{L_{1}, L_{2}}$. A morphism in $2 \mathbf{C o b}^{L_{1}, L_{2}}$ from the empty manifold to itself is given by a compact, oriented, closed 2-manifold $X$ together with a pair of complex line bundles $L_{1} \oplus L_{2} \rightarrow X$.

The category $2 \mathbf{C o b}^{L_{1}, L_{2}}$ is generated by the following finite set of morphisms [4]:
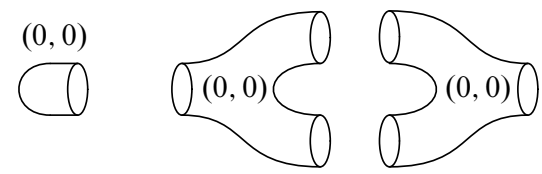

$(0,0)$
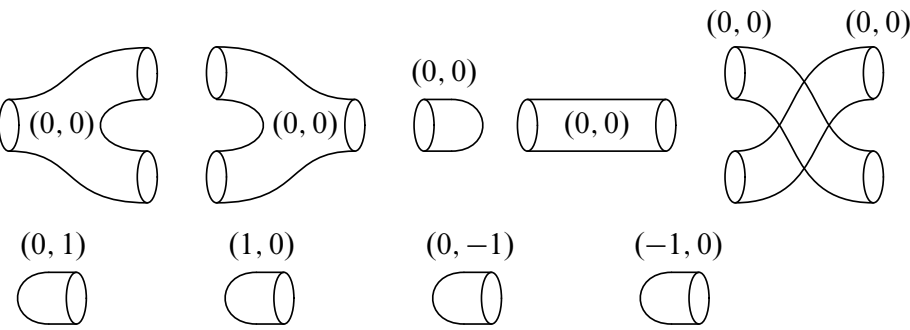


\subsection{The functor DT(-)}

Let $R$ be the ring of Laurent series in $q$ with coefficients given by rational functions in $t_{1}$ and $t_{2}$,

$$
R=\mathbb{Q}\left(t_{1}, t_{2}\right)((q)) .
$$

The collection of partition functions $\mathrm{DT}\left(g \mid k_{1}, k_{2}\right)_{\lambda^{1}, \ldots, \lambda^{r}}$ of degree $d$ gives rise to a functor

$$
\text { DT(-): } 2 \mathbf{C o b}^{L_{1}, L_{2}} \rightarrow R \bmod
$$

as follows. Define

$$
\mathbf{D T}\left(S^{1}\right)=H=\bigoplus_{\lambda \vdash d} R e_{\lambda}
$$

to be the free $R$-module with basis $\left\{e_{\lambda}\right\}_{\lambda \vdash d}$ labelled by partitions of $d$, and let

$$
\text { DT }\left(S^{1} \coprod \cdots \coprod S^{1}\right)=H \otimes \cdots \otimes H \text {. }
$$

Let $W_{s}^{t}\left(g \mid k_{1}, k_{2}\right)$ be the connected genus $g$ cobordism from a disjoint union of $s$ circles to a disjoint union of $t$ circles, equipped with lines bundles $L_{1}$ and $L_{2}$ of level $\left(k_{1}, k_{2}\right)$. We define the $R$-module homomorphism

$$
\text { DT }\left(W_{s}^{t}\left(g \mid k_{1}, k_{2}\right)\right): H^{\otimes s} \rightarrow H^{\otimes t}
$$

by

$$
e_{\eta^{1}} \otimes \cdots \otimes e_{\eta^{s}} \mapsto \sum_{\mu^{1} \ldots \mu^{t} \vdash d} \mathrm{DT}\left(g \mid k_{1}, k_{2}\right)_{\eta^{1}, \ldots, \eta^{s}}^{\mu^{1}, \ldots, \mu^{t}} e_{\mu^{1}} \otimes \cdots \otimes e_{\mu^{t}} .
$$

We extend the definition of DT(-) to disconnected cobordisms by tensor product:

$$
\mathbf{D T}(W[1] \coprod \cdots \coprod W[n])=\mathbf{D T}(W[1]) \otimes \cdots \otimes \mathbf{D T}(W[n]) .
$$

Proposition 3 DT(-): $2 \mathbf{C o b}^{L_{1}, L_{2}} \rightarrow R \bmod$ is a well-defined functor.

Proof The degeneration formula of Donaldson-Thomas theory (see Maulik et al [25]) implies the following compatibility:

$$
\mathbf{D T}\left(\left(W, L_{1}, L_{2}\right) \circ\left(W^{\prime}, L_{1}^{\prime}, L_{2}^{\prime}\right)\right)=\mathbf{D T}\left(W, L_{1}, L_{2}\right) \circ \mathbf{D T}\left(W^{\prime}, L_{1}^{\prime}, L_{2}^{\prime}\right) .
$$

We must also prove DT(-) takes identity morphisms to identity morphisms. Since the tube $W_{1}^{1}(0 \mid 0,0)$ is the identity morphism from $S^{1}$ to $S^{1}$ in $2 \mathbf{C o b}^{L_{1}, L_{2}}$, we require

$$
\mathrm{DT}(0 \mid 0,0)_{\mu}^{v}=\delta_{\mu}^{v} .
$$

Equation (8) is proven in Lemma 4 below. 


\section{Lemma $4 \mathrm{DT}(0 \mid 0,0)_{\mu}^{v}=\delta_{\mu}^{v}$.}

Proof Let $N=\mathcal{O}_{\mathbf{P}^{1}} \oplus \mathcal{O}_{\mathbf{P}^{1}}$ be the trivial bundle with level $(0,0)$ splitting on $\mathbf{P}^{1}$. The moduli space $I_{d}\left(N / N_{0} \cup N_{\infty}, d\right)$ is isomorphic to the Hilbert scheme $\operatorname{Hilb}\left(\mathbb{C}^{2}, d\right)$. The $q$-constant terms of $\mathrm{DT}(0 \mid 0,0)_{\mu, \nu}$ are therefore determined by the intersection form in the Nakajima basis:

$$
\mathrm{DT}(0 \mid 0,0)_{\mu, \nu}=\frac{1}{\left(t_{1} t_{2}\right)^{\ell(\mu)}} \frac{(-1)^{d-\ell(\mu)}}{\mathfrak{z}(\mu)} \delta_{\mu, \nu} .
$$

Hence, the Lemma is proven for $q$-constant terms.

The degeneration formula in Donaldson-Thomas theory yields the following factorization

$$
\sum_{\nu} \mathrm{DT}(0 \mid 0,0)_{\mu}^{\nu} \mathrm{DT}(0 \mid 0,0)_{\nu}^{\rho}=\mathrm{DT}(0 \mid 0,0)_{\mu}^{\rho} .
$$

Since the matrix $\operatorname{DT}(0 \mid 0,0)_{\mu}^{\nu}$ is invertible by the $q$-constant analysis, $\operatorname{DT}(0 \mid 0,0)_{\mu}^{\nu}$ must be the identity matrix (with no $q$-dependence).

\section{Vanishing}

\subsection{Summary}

Consider the local Donaldson-Thomas theory of level $(0,0)$ on a nonsingular curve $C$. Let

$$
T=\mathbb{C}^{*} \times \mathbb{C}^{*}
$$

be the 2-dimensional scaling torus. Let $T^{ \pm}$denote the 1-dimensional anti-diagonal subtorus,

$$
T^{ \pm}=\left\{\left(\xi, \xi^{-1}\right) \mid \xi \in \mathbb{C}^{*}\right\} \subset T .
$$

The $T^{ \pm}$-equivariant Donaldson-Thomas invariants are obtained from the $T$-equivariant invariants by the substitution

$$
t_{1}=t, \quad t_{2}=-t,
$$

where $t$ is the weight of the standard representation of $T^{ \pm}$. We prove vanishing results for the $T^{ \pm}$-equivariant Donaldson-Thomas invariants of level $(0,0)$. 


\subsection{Descendent insertions}

Let $N$ be a split rank 2 bundle on a nonsingular curve $C$ with a scaling $T$-action. We will consider the local Donaldson-Thomas theory of $N$ with descendent insertions. We review the definitions of Maulik et al [25].

The moduli space $I_{n}(N, d)$ is canonically isomorphic to the Hilbert scheme of curves of $N$. Let $\pi_{1}$ and $\pi_{2}$ denote the projections to the respective factors of $I_{n}(N, d) \times N$. Consider the universal ideal sheaf $\mathfrak{I}$,

$$
\mathfrak{I} \rightarrow I_{n}(N, d) \times N .
$$

Since $\mathfrak{I}$ is $\pi_{1}$-flat and $N$ is nonsingular, a finite resolution of $\mathfrak{I}$ by locally free sheaves on $I_{n}(N, d) \times N$ exists. Hence, the Chern classes of $\mathfrak{I}$ are well-defined.

For $\gamma \in A_{T}^{l}(N, \mathbb{Q})$, let $\operatorname{ch}_{k+2}(\gamma)$ denote the following operation on the Chow homology of $I_{n}(N, d)$ :

$$
\begin{aligned}
& \operatorname{ch}_{k+2}(\gamma): A_{*}^{T}\left(I_{n}(N, d), \mathbb{Q}\right) \rightarrow A_{*-k+1-l}^{T}\left(I_{n}(N, d), \mathbb{Q}\right), \\
& \operatorname{ch}_{k+2}(\gamma)(\xi)=\pi_{1 *}\left(\operatorname{ch}_{k+2}(\mathfrak{I}) \cdot \pi_{2}^{*}(\gamma) \cap \pi_{1}^{*}(\xi)\right) .
\end{aligned}
$$

Though $\pi_{1}$ is not proper, the $T$-equivariant push-forward $\pi_{1 *}$ is well-defined by localization.

Descendent fields in Donaldson-Thomas theory, denoted by $\sigma_{k}(\gamma)$, correspond to the operations $\operatorname{ch}_{k+2}(\gamma)$. The $T$-equivariant descendent invariants are defined by

$$
\left\langle\left.\sigma_{k_{1}}\left(\gamma_{l_{1}}\right) \cdots \sigma_{k_{r}}\left(\gamma_{l_{r}}\right)\right|_{n, d} ^{N}=\int_{\left[I_{n}(N, d)^{T}\right]^{\mathrm{vir}}} \frac{\prod_{i=1}^{r} \operatorname{ch}_{k_{i}+2}\left(\gamma_{l_{i}}\right)}{e\left(\text { Norm }^{\mathrm{vir}}\right)},\right.
$$

where the latter integral is the push-forward to a point of the class

$$
\operatorname{ch}_{k_{1}+2}\left(\gamma_{l_{1}}\right) \circ \cdots \circ \operatorname{ch}_{k_{r}+2}\left(\gamma_{l_{r}}\right)\left(\frac{\left[I_{n}(N, d)^{T}\right]^{\text {vir }}}{e\left(\text { Norm }^{\text {vir }}\right)}\right) .
$$

The descendent invariants of $N$ may be viewed equivalently as equivariant residues:

$$
\left\langle\left.\sigma_{k_{1}}\left(\gamma_{l_{1}}\right) \cdots \sigma_{k_{r}}\left(\gamma_{l_{r}}\right)\right|_{n, d} ^{N}=\operatorname{Res}_{I_{n}(N, d)^{T}}\left[\int_{\left[I_{n}(N, d)\right]^{\mathrm{vir}}} \prod_{i=1}^{r} \operatorname{ch}_{k_{i}+2}\left(\gamma_{l_{i}}\right)\right] .\right.
$$

The definition of $T$-equivariant descendent invariants in relative Donaldson-Thomas theory of $N$ is identical. The boundary condition over a relative point $p_{i} \in C$ is determined by a partition $\eta^{i}$ weighted by $H_{T}^{*}\left(N_{p_{i}}, \mathbb{Q}\right)$.

Brackets with relative conditions on the right will often be used. For example,

$$
\left\langle\sigma_{k_{1}}\left(\gamma_{l_{1}}\right) \cdots \sigma_{k_{r}}\left(\gamma_{l_{r}}\right) \mid v^{1}, \ldots, v^{s}\right\rangle_{n, d}^{N}
$$


denotes a descendent invariant relative to $s$ points of $C$.

\subsection{Brackets}

Efficient bracket notation for Donaldson-Thomas invariants will be used throughout the paper.

For absolute brackets (without relative conditions), the degree subscript $d$ is always required. If the Euler characteristic subscript $n$ is omitted, a sum is signified,

$$
\left\langle\prod_{i} \sigma_{k_{i}}\left(\gamma_{l_{i}}\right)\right\rangle_{d}^{N}=\sum_{n} q^{n}\left\langle\prod_{i} \sigma_{k_{i}}\left(\gamma_{l_{i}}\right)\right\rangle_{n, d}^{N} .
$$

If a relative condition occurs in a bracket, the degree subscript is redundant and therefore may be omitted,

$$
\left\langle\prod_{i} \sigma_{k_{i}}\left(\gamma l_{i}\right) \mid v\right\rangle_{n}^{N}=\left\langle\prod_{i} \sigma_{k_{i}}\left(\gamma_{l_{i}}\right) \mid \nu\right\rangle_{n,|v|}^{N} .
$$

If all subscripts in a relative bracket are omitted, a sum is signified,

$$
\left\langle\prod_{i} \sigma_{k_{i}}\left(\gamma_{l_{i}}\right) \mid v\right\rangle^{N}=\sum_{n} q^{n}\left\langle\prod_{i} \sigma_{k_{i}}\left(\gamma_{l_{i}}\right) \mid v\right\rangle_{n,|v|}^{N},
$$

as in the absolute case.

Most of our Donaldson-Thomas computations will concern the local theory of $\mathbf{P}^{1}$. If the superscript $N$ is replaced by a level $\left(k_{1}, k_{2}\right)$, the theory of $\mathbf{P}^{1}$ is signified,

$$
\left\langle\prod_{i} \sigma_{k_{i}}\left(\gamma_{l_{i}}\right) \mid v\right\rangle^{\left(m_{1}, m_{2}\right)}=\left\langle\prod_{i} \sigma_{k_{i}}\left(\gamma_{l_{i}}\right) \mid v\right\rangle^{\mathcal{O}_{\mathbf{P}^{1}}\left(m_{1}\right) \oplus \mathcal{O}_{\mathbf{P} 1}\left(m_{2}\right)} .
$$

If the superscript is omitted altogether, then the level $(0,0)$ theory of $\mathbf{P}^{1}$ is signified,

$$
\left\langle\prod_{i} \sigma_{k_{i}}\left(\gamma_{l_{i}}\right) \mid v\right\rangle=\left\langle\prod_{i} \sigma_{k_{i}}\left(\gamma_{l_{i}}\right) \mid v\right\rangle^{(0,0)} \text {. }
$$

Of course, redundant labels may be kept in various formulas for emphasis.

\subsection{Restriction to $T^{ \pm}$}

Consider the level $(0,0)$ theory on a nonsingular curve $C$. Since the $T^{ \pm}$-fixed locus of $I_{n}\left(\mathcal{O}_{C} \oplus \mathcal{O}_{C}, d\right)$ is proper, the $T^{ \pm}$-equivariant descendent invariants are well-defined by residues. The restriction of the $T$-equivariant descendent invariants to $T^{ \pm}$yield the $T^{ \pm}$-equivariant descendent invariants. The restriction to $T^{ \pm}$can also be seen to be well-defined by the following more precise result. 
Lemma 5 The relative $T$-equivariant descendent invariants of level $(0,0)$ on $C$ take values in the subring

$$
\mathbb{Q}\left[t_{1}, t_{2}\right]_{\left(t_{1} t_{2}\right)} \subset \mathbb{Q}\left(t_{1}, t_{2}\right)
$$

Proof Let $N=\mathcal{O}_{C} \oplus \mathcal{O}_{C}$. As before, let

$$
\mathfrak{J} \rightarrow \mathcal{N}
$$

denote the universal ideal sheaf over the universal total space

$$
\mathcal{N} \rightarrow I_{n}(N / S, d) .
$$

Since $N=C \times \mathbb{C}^{2}$, there is a proper morphism

$$
\rho: \mathcal{N} \rightarrow I_{n}(N / S, d) \times \mathbb{C}^{2} .
$$

Moreover, $\rho_{*}\left(\mathcal{O}_{\mathcal{N}} / \mathfrak{J}\right)$ is flat family over $I_{n}(N / S, d)$ of torsion sheaves of $\mathbb{C}^{2}$ of length $n$ if the genus of $C$ is 0 . There is an associated morphism of Hilbert-Chow type,

$$
\iota: I_{n}(N / S, d) \rightarrow \operatorname{Sym}^{n}\left(\mathbb{C}^{2}\right) .
$$

If the genus $g_{C}>0$, a parallel construction yields

$$
\iota: I_{n}(N / S, d) \rightarrow \operatorname{Sym}^{n+d g_{C}}\left(\mathbb{C}^{2}\right) .
$$

A $T$-equivariant, proper morphism,

$$
\iota^{\prime}: \operatorname{Sym}^{n+d g_{C}}\left(\mathbb{C}^{2}\right) \rightarrow \oplus_{1}^{n+d g_{C}}\left(\mathbb{C}^{2}\right),
$$

is obtained via the higher moments,

$$
\iota^{\prime}\left(\left\{\left(x_{i}, y_{i}\right)\right\}\right)=\left(\sum_{i} x_{i}, \sum_{i} y_{i}\right) \oplus\left(\sum_{i} x_{i}^{2}, \sum_{i} y_{i}^{2}\right) \oplus \cdots \oplus\left(\sum_{i} x_{i}^{n+d g_{c}}, \sum_{i} y_{i}^{n+d g_{c}}\right) .
$$

Let $j=\iota^{\prime} \circ \iota$.

Since $j$ is a $T$-equivariant, proper morphism, there is $T$-equivariant push-forward

$$
j_{*}: A_{*}^{T}\left(I_{n}(N / S, d), \mathbb{Q}\right) \rightarrow A_{*}^{T}\left(\oplus_{1}^{n+d g_{C}}\left(\mathbb{C}^{2}\right), \mathbb{Q}\right) .
$$

Descendent invariants are defined via the $T$-equivariant residue of

$$
\left(\prod_{i} \operatorname{ch}_{k_{i}}\left(\gamma_{l_{i}}\right) \cup \epsilon_{\mathrm{rel}}\right) \cap\left[I_{n}(N / S, d)\right]^{\mathrm{vir}} \in A_{*}^{T}\left(I_{n}(N / S, d), \mathbb{Q}\right),
$$


where $\epsilon_{\text {rel }}$ denotes the relative conditions. We may instead calculate the $T$-equivariant residue of

$$
j_{*}\left(\left(\prod_{i} \operatorname{ch}_{k_{i}}\left(\gamma_{l_{i}}\right) \cup \epsilon_{\mathrm{rel}}\right) \cap\left[I_{n}(N / S, d)\right]^{\mathrm{vir}}\right) \in A_{*}^{T}\left(\oplus_{1}^{n+d g_{C}}\left(\mathbb{C}^{2}\right), \mathbb{Q}\right) .
$$

Since the space $\oplus_{1}^{n+d g_{C}}\left(\mathbb{C}^{2}\right)$ has a unique $T$-fixed point with tangent weights,

$$
t_{1}, t_{2}, 2 t_{1}, 2 t_{2}, \ldots,\left(n+d g_{C}\right) t_{1},\left(n+d g_{C}\right) t_{2},
$$

we conclude the descendent invariant has only monomial poles in the variables $t_{1}$ and $t_{2}$.

We denote the restriction of the $T$-equivariant descendent invariants to the anti-diagonal subtorus by an additional superscript \pm . For example, the restriction of (10) is denoted by

$$
\left\langle\sigma_{k_{1}}\left(\gamma_{l_{1}}\right) \cdots \sigma_{k_{r}}\left(\gamma_{l_{r}}\right) \mid v^{1}, \ldots, v^{s}\right\rangle_{n, d}^{N \pm}
$$

\subsection{Absolute $\mathbf{P}^{1}$}

The bundle $N=\mathcal{O}_{\mathbf{P}^{1}} \oplus \mathcal{O}_{\mathbf{P}^{1}}$ admits a natural action of the 3-dimensional torus,

$$
\mathbf{T}=S \times T,
$$

via the canonical lifting of $S$, see Section 2.2. The $T$-equivariant descendent invariants of $N$ can be calculated by localization on $I_{n}(N, d)$ with respect to the $\mathbf{T}$-action.

The localization of the virtual class $\left[I_{n}(N, d)\right]^{\text {vir }}$ to the $\mathbf{T}$-fixed points of $I_{n}(N, d)$ is determined by the formulas of Maulik et al $[24 ; 25]$ in terms of vertex and edge terms.

Consider first the vertex terms. Let $\Pi(\lambda, \varnothing, \varnothing)$ be the set of 3-dimensional partitions with outgoing 2-dimensional partitions $\lambda, \varnothing$, and $\varnothing$. The partitions $\pi \in \Pi(\lambda, \varnothing, \varnothing)$ are finite in two of the three outgoing directions. The generating series $W(\lambda, \varnothing, \varnothing)$ is defined by

$$
\mathrm{W}(\lambda, \varnothing, \varnothing)=\sum_{\pi \in \Pi(\lambda, \varnothing, \varnothing)} \mathrm{w}(\pi) q^{|\pi|} .
$$

Here, $w(\pi)$ is the equivariant vertex measure, and $|\pi|$ is the number of boxes of $\pi$ which remain after removing the infinite outgoing cylinder [24;25].

Lemma 6 For $\pi \in \Pi(\lambda, \varnothing, \varnothing)$ satisfying $|\pi|>0$, the measure $\left.w(\pi)\right|_{s, t_{1}, t_{2}}$ is divisible by $t_{1}+t_{2}$. 
Proof The proof exactly follows the derivation of [25, Lemma 4]. We determine here the precise positive power of $t_{1}+t_{2}$ dividing the measure $\left.w(\pi)\right|_{s, t_{1}, t_{2}}$.

Let $\lambda$ and $\mu$ be two partition diagrams satisfying $\lambda \supset \mu$. The difference $\lambda / \mu$ between $\lambda$ and $\mu$ is a skew diagram. The content $c(\square)$ of a square of a partition diagram with coordinates $(i, j)$ is defined by

$$
c(\square)=j-i .
$$

A rim hook is a connected skew diagram which does not contain two squares of equal content.

For any skew diagram $\lambda / \mu$ there is a minimal integer $r$ for which

$$
\mu=v_{0} \subset v_{1} \subset \cdots \subset v_{r}=\lambda
$$

and each $v_{k+1} / v_{k}$ is a rim hook. The minimal $r$ is the rank of $\lambda / \mu$. The rank can be determined by repeatedly peeling off maximal rim hooks from $\lambda$. The process can be seen in a rank 4 example:

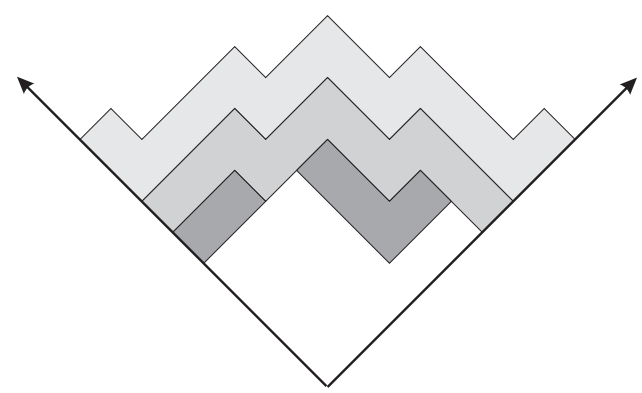

Let $a_{k}$ be the number of squares in $\lambda / \mu$ of content $k$. The rank is determined in terms of $a_{k}$ by

$$
\operatorname{rank} \lambda / \mu=\frac{1}{2} \sum_{k}\left|a_{k}-a_{k+1}\right|
$$

since the right side of (11) receives contributions from the beginning and end of every rim hook. Since each summand in (11) is either 0 or 1 , each term can be squared

$$
\operatorname{rank} \lambda / \mu=\frac{1}{2} \sum_{k}\left(a_{k}-a_{k+1}\right)^{2} \text {. }
$$

A 3-dimensional partition with one leg of profile $\lambda$ can be viewed as a sequence of slices by planes perpendicular to the direction of the leg:

$$
\lambda^{0} \supset \lambda^{(1)} \supset \lambda^{(2)} \supset \lambda^{(M)}=\cdots=\lambda^{(\infty)}=\lambda, \quad M \gg 0 .
$$


The order of divisibility of $\left.w(\pi)\right|_{s, t_{1}, t_{2}}$ by $t_{1}+t_{2}$ is

$$
\left.\operatorname{ord}_{\left(t_{1}+t_{2}\right)} \mathrm{w}(\pi)\right|_{s, t_{1}, t_{2}}=\sum_{k=0}^{\infty} \operatorname{rank} \lambda^{(k)} / \lambda^{(k+1)},
$$

where, in fact, only finitely many terms are nonzero. Formula (13) is a direct consequence of (12) and the proof of [25, Lemma 4].

The $\mathbf{T}$-fixed points of $I_{n}(N, d)$ are isolated and correspond bijectively to triples $\left(\pi, \lambda, \pi^{\prime}\right)$ where $\pi, \pi^{\prime} \in \Pi(\lambda, \varnothing, \varnothing)$ and

$$
|\pi|+\left|\pi^{\prime}\right|=n-|\lambda| \text {. }
$$

The vertex partitions $\pi, \pi^{\prime}$ determine the nonreduced structure of the $\mathbf{T}$-fixed ideal over $0, \infty \in \mathbf{P}^{1}$. The edge partition $\lambda$ determines the nonreduced structure over $\mathbf{P}^{1} \backslash\{0, \infty\}$. The $\mathbf{T}$-equivariant localization of the virtual class $\left[I_{n}(N, d)\right]^{\mathrm{vir}}$ to the fixed point $\left(\pi, \lambda, \pi^{\prime}\right)$ is

$$
\left.\left.\mathrm{w}(\pi)\right|_{s, t_{1}, t_{2}} \cdot E(\lambda) \cdot \mathrm{w}\left(\pi^{\prime}\right)\right|_{-s, t_{1}, t_{2}},
$$

where the edge terms $E(\lambda)$ are the (inverse) tangent $T$-weights to $\operatorname{Hilb}\left(\mathbb{C}^{2},|\lambda|\right)$ at the $T$-fixed point indexed by $\lambda$. The edge terms are easily seen to be prime to $t_{1}+t_{2}$. By Lemma 6, the localization (14) vanishes when restricted to the anti-diagonal torus $T^{ \pm}$if either $|\pi|$ or $\left|\pi^{\prime}\right|$ are positive.

Lemma 7 For $n>d$,

$$
\left\langle\left.\sigma_{k_{1}}\left(\gamma_{l_{1}}\right) \cdots \sigma_{k_{r}}\left(\gamma_{l_{r}}\right)\right|_{n, d} ^{\mathcal{O}_{\mathbf{p}^{1}} \oplus \mathcal{O}_{\mathbf{p}^{1}} \pm}=0\right.
$$

Proof The result is a consequence of the vanishing (14) of the virtual class when localized to the $\mathbf{T}$-fixed points (and restricted to $T^{ \pm}$). The descendent integrand plays no role.

Lemma 7 is the first vanishing result for the $T^{ \pm}$-equivariant Donaldson-Thomas invariants of level $(0,0)$.

\subsection{The matrix $M_{d}$}

Let $N=\mathcal{O}_{\mathbf{P}^{1}} \oplus \mathcal{O}_{\mathbf{P}^{1}}$ be the trivial bundle with level $(0,0)$ splitting over $\mathbf{P}^{1}$. Let $N_{0}$ denote the fiber of $N$ over $0 \in \mathbf{P}^{1}$. Let

$$
\left[N_{0}\right] \in A_{T}^{1}(N, \mathbb{Q})
$$


be the associated class.

We define a matrix $M_{d}$ of descendent invariants of $N$ relative to $N_{\infty}$ indexed by the set $\mathcal{P}(d)$ of partitions of $d$. For partitions $\mu, v \in \mathcal{P}(d)$, let

$$
M_{d}(\mu, v)=q^{-d}\left\langle\prod_{i} \sigma_{\mu_{i}-1}\left(\left[N_{0}\right]\right) \mid v([0])\right\rangle^{N},
$$

following the bracket conventions of Section 4.3. The partition $\mu$ specifies a descendent insertion, and the partition $v$ specifies a relative condition along $N_{\infty}$. Since, the minimal Euler characteristic of a degree $d$ subscheme of $N$ is $d$, the elements of $M_{d}$ lie in the ring $\mathbb{Q}\left[t_{1}, t_{2}\right]_{\left(t_{1} t_{2}\right)} \llbracket q \rrbracket$.

Define the length partial order on $\mathcal{P}(d)$ by the following rule: $\mu \geq \mu^{\prime}$ if $\ell(\mu)>\ell\left(\mu^{\prime}\right)$ or if $\mu=\mu^{\prime}$.

Lemma $8 M_{d}$ is upper triangular with respect to the length partial ordering.

Proof Let $N \subset \bar{N}$ denote the $T$-equivariant compactification over $\mathbf{P}^{1}$ defined by

$$
\bar{N}=\mathbf{P}\left(N \oplus \mathcal{O}_{\mathbf{P}^{1}}\right) \text {. }
$$

Here, $T$ acts trivially on the additional $\mathcal{O}_{\mathbf{p}^{1}}$. We will only consider the curve classes on $\bar{N}$ obtained from $N$.

Let $\bar{N}_{\infty}$ denote the fiber of the compactification over $\infty \in \mathbf{P}^{1}$. Let

$$
0_{\uparrow}, 0_{\rightarrow} \in \bar{N}_{\infty}
$$

be the two new $T$-fixed points with normal $T$-weights

$$
t_{1}-t_{2},-t_{2} \text {, and } t_{2}-t_{1},-t_{1}
$$

respectively.

By the residue definition, the $T$-equivariant Donaldson-Thomas descendent invariant

$$
\left\langle\prod_{i} \sigma_{\mu_{i}-1}\left(\left[N_{0}\right]\right) \mid v([0])\right\rangle_{n}^{N}
$$

occurs as a summand in the localization computation of the $T$-equivariant descendent

$$
\left\langle\prod_{i} \sigma_{\mu_{i}-1}\left(\left[\bar{N}_{0}\right]\right) \mid v([0])\right\rangle_{n}^{\bar{N}}
$$

for any partition $\mu-$ not necessarily a partition of $d$. 
The virtual dimension of the moduli space $I_{n}\left(\bar{N} / \bar{N}_{\infty}, d\right)$ is $2 d$. The integrand and relative constraints of (16) impose

$$
|\mu|-\ell(\mu)+d+\ell(v)
$$

conditions. Therefore, since $\bar{N}$ is proper, the integral (16) vanishes if

$$
|\mu|-\ell(\mu)+\ell(v)<d .
$$

The $T$-localization formula expresses (16) as the following sum of triple products of relative local Donaldson-Thomas invariants of $N$ :

$$
\begin{gathered}
\sum_{n_{0}+n_{\uparrow}+n_{\rightarrow}=n} \sum_{A_{0} \cup A_{\uparrow} \cup A \rightarrow=\{1, \ldots, \ell(\mu)\}}\left\langle\prod_{i \in A_{0}} \sigma_{\mu_{i}-1}\left(\left[N_{0}\right]\right)|v([0])|_{n_{0}}^{N}\right. \\
\left.\left.\cdot\left\langle\prod_{i \in A_{\uparrow}} \sigma_{\mu_{i}-1}\left(\left[N_{0}\right]\right) \mid \varnothing\right\rangle_{n_{\uparrow}}^{N}\right|_{t_{1}-t_{2},-t_{2}} \cdot\left\langle\prod_{i \in A_{\rightarrow}} \sigma_{\mu_{i}-1}\left(\left[N_{0}\right]\right) \mid \varnothing\right\rangle_{n_{\rightarrow}}^{N}\right|_{t_{2}-t_{1},-t_{1}} .
\end{gathered}
$$

The relative localization formula is applied with a factorization rule - the $T$-fixed loci do not geometrically factor. ${ }^{3}$

By induction on $n$ and $\ell(\mu)$, we conclude

$$
\left\langle\prod_{i} \sigma_{\mu_{i}-1}\left(\left[N_{0}\right]\right) \mid v([0])\right\rangle_{n}^{N}=0
$$

if $|\mu|-\ell(\mu)+\ell(\nu)<d$. In particular,

$$
M_{d}(\mu, v)=0
$$

if $\ell(\mu)>\ell(\nu)$.

If $|\mu|-\ell(\mu)+\ell(v)=d$, the constraints of (16) impose exactly $2 d$ conditions. The nonequivariant integral

$$
\left\langle\prod_{i} \sigma_{\mu_{i}-1}\left(\left[\bar{N}_{0}\right]\right) \mid v_{1}([\gamma]), v_{2}([0]), \ldots, v_{\ell(v)}([0])\right\rangle_{n}^{\bar{N}}
$$

is independent of $\gamma \in \bar{N}_{\infty}$ - the parts of the relative condition over $\infty$ are written explicitly here. After specializing $\gamma$ to a $T$-fixed point of $\bar{N}_{\infty}$, the invariant (19) can be computed by localization with respect to $T$.

\footnotetext{
${ }^{3}$ In the absolute case, the $T$-fixed loci of $I_{n}(\bar{N}, d)$ factor as a triple product. The $T$-fixed loci relative to a fiber, $I_{n}\left(\bar{N} / \bar{N}_{\infty}, d\right)$ do $n o t$ factor. However, a factorization rule holds. Factorization can be deduced from the relative localization formula applied to disjoint unions following the discussion of connected/ disconnected issues in relative Gromov-Witten theory in Maulik-Pandharipande [26, Section 1.8].
} 
If $\gamma$ is specialized to $0 \in \bar{N}_{\infty}, T$-equivariant localization expresses (19), as before, as a sum of triple product (17). The vanishing (18) removes most terms.

If $\gamma$ is specialized to $0 \uparrow$, the $T$-equivariant localization formula for (19) takes a different form:

$$
\begin{gathered}
\sum_{n_{0}+n_{\uparrow}+n_{\rightarrow}=n} \sum_{A_{0} \cup A_{\uparrow} \cup A_{\rightarrow}=\{1, \ldots, \ell(\mu)\}}\left\langle\prod_{i \in A_{0}} \sigma_{\mu_{i}-1}\left(\left[N_{0}\right]\right) \mid v_{2}([0]), \ldots, v_{\ell(v)}([0])\right\rangle_{n_{0}}^{N} \\
\left.\cdot\left\langle\prod_{i \in A_{\uparrow}} \sigma_{\mu_{i}-1}\left(\left[N_{0}\right]\right) \mid v_{1}([0])\right\rangle_{n_{\uparrow}}^{N}\right|_{t_{1}-t_{2},-t_{2}} \cdot\left\langle\left.\prod_{i \in A_{\rightarrow}} \sigma_{\mu_{i}-1}\left(\left[N_{0}\right]\right)|\varnothing|_{n_{\rightarrow}}^{N}\right|_{t_{2}-t_{1},-t_{1}} .\right.
\end{gathered}
$$

By repeated use of the comparison of the two evaluations of (19), we find

$$
\left\langle\prod_{i} \sigma_{\mu_{i}-1}\left(\left[N_{0}\right]\right) \mid v([0])\right\rangle_{n}^{N}=0
$$

unless there are disjoint subpartitions $\mu[i] \subset \mu$ such that

$$
|\mu[i]|-\ell(\mu[i])+1=v_{i} .
$$

If $\mu \in \mathcal{P}(d)$ and $\ell(\mu)=\ell(\mu)$, condition (21) implies $\mu=v$. Hence,

$$
M_{d}(\mu, v)=0
$$

if $\ell(\mu)=\ell(v)$ unless $\mu=v$.

Lemma $9 M_{d}$ is invertible in the ring of matrices with $\mathbb{Q}\left[t_{1}, t_{2}\right]_{\left(t_{1} t_{2}\right)} \llbracket q \rrbracket$ coefficients.

Proof The minimal Euler characteristic of a degree $d$ subscheme of $N$ is $d$. Since

$$
I_{d}(N, d) \cong \operatorname{Hilb}\left(N_{\infty}, d\right),
$$

the matrix of $q$-constant terms of $M_{d}$ is determined by the classical (equivariant) intersection theory of the Hilbert scheme of points of the plane and is well-known to be invertible.

Let $M_{d}^{ \pm}$denote the restriction of $M_{d}$ to the anti-diagonal torus. The following vanishing result holds.

Lemma $10 M_{d}^{ \pm}$has no $q$ dependence. 
Proof Let $C_{d}$ be a matrix indexed by partitions $\mathcal{P}(d)$ with the coefficients

$$
C_{d}(\mu, v)=q^{-d}\left\langle\prod_{i} \sigma_{\mu_{i}-1}\left(\left[N_{0}\right]\right) \cdot \prod_{j} \sigma_{\nu_{j}-1}\left(\left[N_{\infty}\right]\right)\right\rangle_{d}^{N} .
$$

The degeneration formula in Donaldson-Thomas theory, yields the factorization

$$
M_{d} \triangle_{d} M_{d}^{t}=C_{d}
$$

where $\Delta_{d}$ is defined by (5). The matrix $\Delta_{d}$ has no $q$ dependence.

Equation (22) is obtained by a degeneration of the base to a reducible nodal curve:

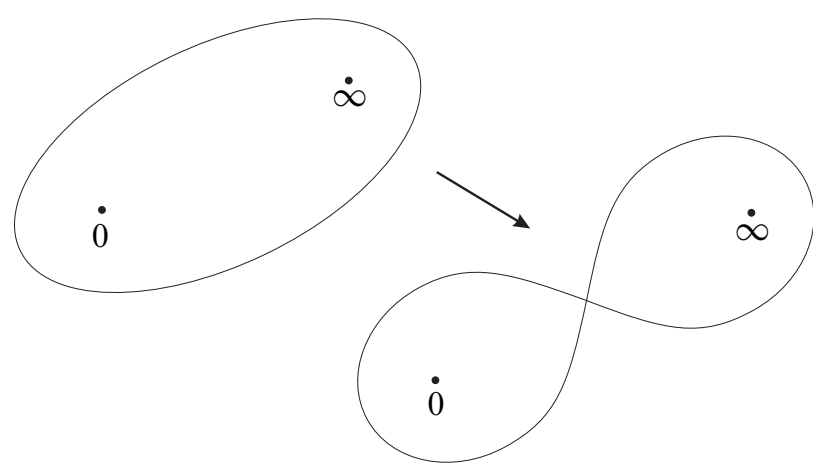

The bundle $N$ specializes to a sum of trivial bundles on the reducible curve. The degeneration is equivariant for the scaling torus $T$.

The restriction $C_{d}^{ \pm}$has no $q$ dependence by Lemma 7. The $T^{ \pm}$-restriction of (22) is a Gauss decomposition of $C_{d}^{ \pm}$. Uniqueness of the Gauss decomposition implies the Lemma.

Lemma 10 can be restated as an explicit vanishing of Donaldson-Thomas invariants. For $n>d$,

$$
\left\langle\prod_{i} \sigma_{\mu_{i}-1}\left(\left[N_{0}\right]\right) \mid v([0])\right\rangle_{n, d}^{\mathcal{O}_{\mathbf{p}^{1}} \oplus \mathcal{O}_{\mathbf{p}^{1}} \pm}=0,
$$

where $\mu, v \in \mathcal{P}(d)$. 


\subsection{Degeneration}

Let $N=\mathcal{O}_{C} \oplus \mathcal{O}_{C}$ be the trivial bundle with level $(0,0)$ splitting over a nonsingular, genus $g$ curve $C$. The class

$$
\left[N_{z}\right] \in A_{T}^{1}(N, \mathbb{Q})
$$

is independent of $z \in C$. We will denote the fiber class $\left[N_{z}\right]$ by $F$.

Lemma 11 For $n>d(1-g)$,

$$
\left\langle\prod_{i} \sigma_{k_{i}}(F) \mid v^{1}, \ldots, v^{s}\right\rangle_{n, d}^{\mathcal{O}_{C} \oplus \mathcal{O}_{C} \pm}=0 .
$$

Proof Since the matrix $M_{d}^{ \pm}$is invertible and has no $q$ dependence, the relative conditions in the Donaldson-Thomas integral (24) can be systematically traded for descendent insertions. The Lemma is then equivalent to the vanishing for $n>d(1-g)$ of all absolute invariants

$$
\left\langle\prod_{i^{\prime}} \sigma_{k_{i^{\prime}}^{\prime}}(F)\right\rangle_{n, d}^{\mathcal{O}_{C} \oplus \mathcal{O}_{C} \pm}=0 .
$$

After degenerating $C$ to a nodal rational curve (and again trading relative conditions for descendent insertions), we need only prove the vanishing (25) in case $C$ is $\mathbf{P}^{1}$. The latter vanishing is a consequence of Lemma 7.

\subsection{Cotangent lines, rubber and topological recursion}

Let $N=\mathcal{O}_{C} \oplus \mathcal{O}_{C}$ be the trivial bundle with level $(0,0)$ splitting over a nonsingular curve $C$. Consider the moduli space of ideal sheaves $I_{n}\left(N / N_{p}, d\right)$ relative to the fiber over $p \in C$. The cotangent line bundle,

$$
\mathbb{L}_{p} \rightarrow I_{n}\left(N / N_{p}, d\right),
$$

is defined by the cotangent space at the relative point $p$ of the target curve. The torus $T$ acts trivially on $\mathbb{L}_{p}$. Let

$$
\psi_{p} \in A_{T}^{1}\left(I_{n}\left(N / N_{p}, d\right), \mathbb{Q}\right)
$$

denote the first Chern class of $\mathbb{L}_{p}$.

The Donaldson-Thomas theory of rubber naturally arises at the boundary of the moduli space $I_{n}\left(N / N_{p}, d\right)$. Let $R$ be a rank 2 bundle of level $(0,0)$ over $\mathbf{P}^{1}$. Let

$$
R_{0}, R_{\infty} \subset R
$$


denote the fibers over $0, \infty \in \mathbf{P}^{1}$. The 1 -dimensional torus $S$ acts on $R$ via the symmetries of $\mathbf{P}^{1}$. Let $I_{n}\left(R / R_{0} \cup R_{\infty}, d\right)$ be the relative moduli space of ideal sheaves, and let

$$
I_{n}\left(R / R_{0} \cup R_{\infty}, d\right)^{\circ} \subset I_{n}\left(R / R_{0} \cup R_{\infty}, d\right)
$$

denote the open set with finite stabilizers for the $S$-action and no destabilization over $\infty \in \mathbf{P}^{1}$. The rubber moduli space,

$$
I_{n}\left(R / R_{0} \cup R_{\infty}, d\right)^{\sim}=I_{n}\left(R / R_{0} \cup R_{\infty}, d\right)^{\circ} / S,
$$

denoted by a superscripted tilde, is determined by the (stack) quotient. The moduli space is empty unless $n>d$. The rubber theory of $R$ is defined by integration against the rubber virtual class,

$$
\left[I_{n}\left(R / R_{0} \cup R_{\infty}, d\right)^{\sim}\right]^{\mathrm{vir}} .
$$

All of the above rubber constructions are $T$-equivariant.

The rubber moduli space $I_{n}\left(R / R_{0} \cup R_{\infty}, 0\right)^{\sim}$ carries cotangent lines at the dynamical points 0 and $\infty$ of $\mathbf{P}^{1}$. Let

$$
\psi_{0}, \psi_{\infty} \in A_{T}^{1}\left(I_{n}\left(R / R_{0} \cup R_{\infty}, d\right)^{\sim}, \mathbb{Q}\right)
$$

denote the associated cotangent line classes. Rubber integrals with relative conditions $\mu$ over 0 and $\nu$ over $\infty$ are denoted by the bracket

$$
\left\langle\mu\left|\psi_{0}^{a} \psi_{\infty}^{b}\right| v\right\rangle_{n, d}^{\sim}
$$

Cotangent line classes in Donaldson-Thomas theory of $N$ can be removed via topological recursion relations. For the relative theory of $N / \cup_{i=1}^{s} N_{p_{i}}$, the topological recursion relation is:

$$
\begin{aligned}
& \left\langle\prod_{i} \sigma_{k_{i}}(F) \prod_{j=1}^{s} \psi_{p_{j}}^{a_{j}} \mid v^{1}, \ldots, v^{s}\right\rangle_{n, d}^{N}= \\
& \sum_{|\eta|=d} \sum_{n_{1}+n_{2}=n+d}\left\langle\prod_{i} \sigma_{k_{i}}(F) \prod_{j \neq 1} \psi_{p_{j}}^{a_{j}} \mid \eta, v^{2}, \ldots v^{s}\right\rangle_{n_{1}, d}^{N} \cdot \triangle_{d}(\eta, \eta)\left\langle\eta\left|\psi_{\infty}^{a_{1}-1}\right| v^{1}\right\rangle_{n_{2}, d}^{\sim} .
\end{aligned}
$$

The proof follows from the degeneration formula of Donaldson-Thomas theory applied to the boundary expression for $\psi_{p_{1}}$ on the Artin stack of target destabilizations. The relative conditions away from $p_{1}$ and the descendent insertions are bystanders in the topological recursion relation. 


\subsection{Rubber calculus}

The rubber integrals (26) are determined via a Donaldson-Thomas rubber calculus. The technique, following Gromov-Witten theory (see Maulik-Pandharipande [26] and Okounkov-Pandharipande [30]), involves rigidification and topological recursion.

The universal target over the rubber moduli space is no longer a product. Let

$$
\pi: \mathcal{R} \rightarrow I_{n}\left(R / R_{0} \cup R_{\infty}, d\right)^{\sim}
$$

denote the universal target. The space $\mathcal{R}$ can be viewed as a moduli space of rubber ideal sheaves together with a point $r$ of the target rubber. The point $r$ is not permitted to lie on the relative divisors $R_{0}$ and $R_{\infty}$. The stability condition is given by finiteness of the associated automorphism group. The virtual class of $\mathcal{R}$ is obtained via $\pi$-flat pull-back,

$$
[\mathcal{R}]^{\mathrm{vir}}=\pi^{*}\left(\left[I_{n}\left(R / R_{0} \cup R_{\infty}, d\right)^{\sim}\right]^{\mathrm{vir}}\right) .
$$

As before, let

$$
\mathfrak{J} \rightarrow \mathcal{R}
$$

denote the universal ideal sheaf on $\mathcal{R}$.

The target point $r$ together with $R_{0}$ and $R_{\infty}$ specifies 3 distinct points of the destabilized $\mathbf{P}^{1}$ over which the rubber is fibered. By viewing the target point as $1 \in \mathbf{P}^{1}$, we obtain a rigidification map,

$$
\phi: \mathcal{R} \rightarrow I_{n}\left(N / N_{0} \cup N_{\infty}, d\right),
$$

where $N=\mathcal{O}_{\mathbf{P}^{1}} \oplus \mathcal{O}_{\mathbf{P}^{1}}$ is the trivial bundle with level $(0,0)$ splitting over $\mathbf{P}^{1}$. By a comparison of deformation theories,

$$
[\mathcal{R}]^{\mathrm{vir}}=\phi^{*}\left(\left[I_{n}\left(N / N_{0} \cup N_{\infty}, d\right)\right]^{\mathrm{vir}}\right) .
$$

Rubber calculus transfers rubber integrals (26) to descendent integrals on rigid (nonrubber) targets via the maps $\pi$ and $\phi$. To start,

$$
(d-n)\left[I_{n}\left(R / R_{0} \cup R_{\infty}, d\right)\right]^{\mathrm{vir}}=\pi_{*}\left(\operatorname{ch}_{3}(\mathfrak{J}) \cap[\mathcal{R}]^{\mathrm{vir}}\right)
$$

by a $\pi$-fiberwise calculation. Since $n>d$, for nonempty rubber moduli spaces, $d-n$ is negative. By the push-pull formula,

$$
(d-n)\left\langle\mu\left|\psi_{0}^{a} \psi_{\infty}^{b}\right| v\right\rangle_{n, d}^{\sim}=\left\langle\left.\mu\left|\operatorname{ch}_{3}(\mathfrak{J}) \pi^{*}\left(\psi_{0}^{a} \psi_{\infty}^{b}\right)\right| v\right|_{n, d} ^{\mathcal{R} \sim} .\right.
$$

Next, we compare the cotangent lines $\pi^{*}\left(\psi_{0}\right)$ and $\phi^{*}\left(\psi_{0}\right)$ on $\mathcal{R}$. A standard argument yields:

$$
\pi^{*}\left(\psi_{0}\right)=\phi^{*}\left(\psi_{0}\right)-\phi^{*}\left(D_{0}\right),
$$


where

$$
D_{0} \subset I_{n}\left(N / N_{0} \cup N_{\infty}, d\right)
$$

is the virtual boundary divisor for which the rubber over $\infty$ carries Euler characteristic $n$. Similarly,

$$
\pi^{*}\left(\psi_{\infty}\right)=\phi^{*}\left(\psi_{\infty}\right)-\phi^{*}\left(D_{\infty}\right)
$$

We will apply the cotangent line comparisons to the right side of (28).

Consider the Hilbert scheme of points $\operatorname{Hilb}\left(R_{0}, d\right)$ of the relative divisor. The boundary condition $\mu$ corresponds to a Nakajima basis element of $A_{T}^{*}\left(\operatorname{Hilb}\left(R_{0}, d\right), \mathbb{Q}\right)$. Let $\mathfrak{J}_{0}$ be the universal ideal sheaf on

$$
\operatorname{Hilb}\left(R_{0}, d\right) \times R_{0},
$$

and let

$$
\sigma_{1}=\pi_{*}\left(\operatorname{ch}_{3}\left(\mathfrak{J}_{0}\right)\right) \in A_{T}^{1}\left(\operatorname{Hilb}\left(R_{0}, d\right), \mathbb{Q}\right) .
$$

The class $\sigma_{1}$ in $A_{T}^{1}\left(\operatorname{Hilb}\left(R_{\infty}, d\right)\right.$ is defined in the same way.

The cotangent line comparisons and equation (28) together yield the following result:

$$
\begin{aligned}
& (d-n)\left\langle\mu\left|\psi_{0}^{a} \psi_{\infty}^{b}\right| v\right\rangle_{n, d}^{\sim}= \\
& \left\langle\mu\left|\sigma_{1}(F) \psi_{0}^{a} \psi_{\infty}^{b}\right| v\right\rangle_{n, d}^{N}-\left\langle\sigma_{1} \cdot \mu\left|\psi_{0}^{a-1} \psi_{\infty}^{b}\right| v\right\rangle_{n, d}^{\sim}-\left\langle\left.\mu\left|\psi_{0}^{a} \psi_{\infty}^{b-1}\right| \sigma_{1} \cdot v\right|_{n, d} ^{\sim} .\right.
\end{aligned}
$$

The rubber integrals (26) are expressed in terms of the relative Donaldson-Thomas theory of $N$ with descendent insertions $\sigma_{k}([F])$ by repeated applications of equation (29) and the topological recursion relations.

The following two vanishing statements are a consequence of the rubber calculus and Lemma 11.

Lemma 12 For $n>d(1-g)$,

$$
\left\langle\prod_{i} \sigma_{k_{i}}(F) \prod_{j=1}^{s} \psi_{p_{j}}^{a_{j}} \mid v^{1}, \ldots, v^{s}\right\rangle_{n, d}^{\mathcal{O}_{C} \oplus \mathcal{O}_{C} \pm}=0
$$

Lemma 13 For $n>d$,

$$
\left\langle\mu\left|\psi_{0}^{a} \psi_{\infty}^{b}\right| \nu\right\rangle_{n, d}^{\sim \pm}=0
$$




\subsection{Parallels}

The anti-diagonal vanishing in level $(0,0)$ holds for all vertices in the triangle of equivalences of Section 1.7.

The Gromov-Witten vanishing follows easily from Mumford's Hodge bundle relation

$$
c(\mathbb{E}) \cdot c\left(\mathbb{E}^{\vee}\right)=1
$$

on the moduli space of curves $\bar{M}_{g}$, see Bryan-Pandharipande [4]. The Hilbert scheme vanishing is obtained from the existence of a modified virtual class in the hyperkähler setting. The proof requires a restriction of the obstruction theory of maps to $\operatorname{Hilb}\left(\mathbb{C}^{2}, d\right)$, see Okounkov-Pandharipande [31]. The Donaldson-Thomas vanishing proven here could also be pursued via a construction of a modified virtual class. ${ }^{4}$ Instead, our derivation proceeds formally from the equivariant vertex calculations of Maulik et al $[24 ; 25]$ using localization and degeneration.

\section{Additivity}

\subsection{Summary}

The level $(0,0)$ Donaldson-Thomas theory of $\mathbf{P}^{1}$ relative to $0, \infty \in \mathbf{P}^{1}$ will play a crucial role in the study of local curves. The main results of the Section are vanishing and additivity properties for the invariants

$$
\left\langle\mu\left|\sigma_{1}(F)\right| v\right\rangle
$$

We follow here the bracket conventions of Section 4.3.

The vanishing of the invariants (30) in most cases is established by the following three results.

Lemma 14 If $|\ell(\mu)-\ell(v)|>1$, then $\left\langle\mu\left|\sigma_{1}(F)\right| v\right\rangle$ vanishes.

Lemma 15 If $|\ell(\mu)-\ell(v)|=1$, then $\left\langle\mu\left|\sigma_{1}(F)\right| v\right\rangle_{n, d}$ vanishes for $n>d$.

Lemma 16 If $\ell(\mu)=\ell(v)$, then $\left\langle\mu\left|\sigma_{1}(F)\right| v\right\rangle$ vanishes unless $\mu=v$.

\footnotetext{
${ }^{4}$ Recently, such vanishing arguments have appeared: see Kiem-Li [13] and Maulik-PandharipandeThomas [27].
} 
In the diagonal case, we will obtain the form

$$
\left\langle\mu\left|\sigma_{1}(F)\right| \mu\right\rangle_{n}=\gamma_{\mu, n}\left(t_{1} t_{2}\right)^{-\ell(\mu)}\left(t_{1}+t_{2}\right)
$$

for $\gamma_{\mu, n} \in \mathbb{Q}$ and prove a crucial additivity property parallel to Equation (25) of Okounkov-Pandharipande [31].

Proposition 17 An additivity relation holds:

$$
\frac{\left\langle\mu\left|\sigma_{1}(F)\right| \mu\right\rangle}{\langle\mu \mid \mu\rangle_{|\mu|,|\mu|}}=\sum_{i} q^{|\mu|-\mu_{i}} \frac{\left\langle\mu_{i}\left|\sigma_{1}(F)\right| \mu_{i}\right\rangle}{\left\langle\mu_{i} \mid \mu_{i}\right\rangle_{\mu_{i}, \mu_{i}}}-(\ell(\mu)-1)\left(t_{1}+t_{2}\right) q^{|\mu|} \Phi(q) .
$$

The bracket $\langle\mu \mid \mu\rangle_{|\mu|,|\mu|}$ is the intersection form

$$
\langle\mu \mid \mu\rangle_{|\mu|,|\mu|}=\frac{1}{\left(t_{1} t_{2}\right)^{\ell(\mu)}} \frac{(-1)^{d-\ell(\mu)}}{\mathfrak{z}(\mu)}
$$

in the Nakajima basis. The function $\Phi(q)$ is defined by

$$
\Phi(q)=q \frac{d}{d q} \log M(-q),
$$

where $M(q)$ is the MacMahon series.

\subsection{Proofs of Lemmas 14 and 15}

Let $N=\mathcal{O}_{\mathbf{P}^{1}} \oplus \mathcal{O}_{\mathbf{P}^{1}}$ be the trivial bundle with splitting of level $(0,0)$. Let $\bar{N}$ denote the compactification of $N$ defined in Section 4.6. The proofs are obtained from dimensional analysis for integrals in the Donaldson-Thomas theory of $\bar{N}$.

By linearity, we can trade the invariants with unweighted relative conditions for weighted relative conditions:

$$
\begin{aligned}
\left\langle\mu\left|\sigma_{1}(F)\right| v\right\rangle & =\frac{1}{\left(t_{1} t_{2}\right)^{\ell(\mu)}}\left\langle\mu([0])\left|\sigma_{1}(F)\right| v\right\rangle \\
& =\frac{1}{\left(t_{1} t_{2}\right)^{\ell(v)}}\left\langle\mu\left|\sigma_{1}(F)\right| v([0])\right\rangle
\end{aligned}
$$

By $T$-localization on $\bar{N}$ and the vanishing of Lemma 4, we find

$$
\begin{aligned}
\left\langle\left.\mu([0])\left|\sigma_{1}(F)\right| v\right|_{n} ^{\bar{N}}=\right. & \left\langle\mu([0])\left|\sigma_{1}(F)\right| v\right\rangle_{n} \\
& +q^{d}\langle\mu([0]) \mid v\rangle_{d, d} \cdot\left\langle\left.\left.\varnothing\left|\sigma_{1}(F)\right| \varnothing\right|_{n-d}\right|_{t_{1}-t_{2},-t_{2}}\right. \\
& +\left.q^{d}\langle\mu([0]) \mid v\rangle_{d, d} \cdot\left\langle\varnothing\left|\sigma_{1}(F)\right| \varnothing\right\rangle_{n-d}\right|_{t_{2}-t_{1},-t_{1}}
\end{aligned}
$$


If $1+\ell(\mu)-\ell(v)<0$, then

$$
\left\langle\mu([0])\left|\sigma_{1}(F)\right| \nu\right\rangle_{n}^{\bar{N}}=0
$$

by dimension considerations since $\bar{N}$ is proper. If $\ell(\mu)-\ell(v)<0$, then

$$
\langle\mu([0]) \mid v\rangle_{d, d}=0 .
$$

Therefore, $1+\ell(\mu)-\ell(v)<0$ implies

$$
\left\langle\mu([0])\left|\sigma_{1}(F)\right| v\right\rangle_{n}=0 .
$$

Similarly, if $1-\ell(\mu)+\ell(v)<0$, then

$$
\left\langle\mu\left|\sigma_{1}(F)\right| v([0])\right\rangle_{n}=0 .
$$

We conclude $\left\langle\mu\left|\sigma_{1}(F)\right| \nu\right\rangle$ vanishes unless $|\ell(\mu)-\ell(\nu)| \leq 1$. Lemma 14 is proven.

If the equality $|\ell(\mu)-\ell(v)|=1$ holds, then the argument yields a finer result. Either

$$
\left\langle\mu([0])\left|\sigma_{1}(F)\right| v\right\rangle_{n, d}=\left\langle\mu([0])\left|\sigma_{1}(F)\right| v\right\rangle_{n, d}^{\bar{N}} \in \mathbb{Q}
$$

or

$$
\left\langle\mu\left|\sigma_{1}(F)\right| v([0])\right\rangle_{n, d}=\left\langle\mu\left|\sigma_{1}(F)\right| v([0])\right\rangle_{n, d}^{\bar{N}} \in \mathbb{Q} .
$$

If $n>d$, the factor $\left(t_{1}+t_{2}\right)$ must divide the invariant

$$
\left\langle\mu\left|\sigma_{1}(F)\right| v\right\rangle_{n, d}
$$

by Lemma 11. Thus, if $|\ell(\mu)-\ell(v)|=1$ and $n>d$, the integral (32) must vanish. Lemma 15 is proven.

\subsection{Degree 0 calculation}

We calculate the integral $\left\langle\varnothing\left|\sigma_{1}(F)\right| \varnothing\right\rangle$. By the degeneration formula and Theorem 1 ,

$$
\begin{aligned}
\left\langle\varnothing\left|\sigma_{1}(F)\right| \varnothing\right\rangle & =\frac{\left\langle\sigma_{1}(F)\right\rangle_{0}}{\langle\varnothing \mid\rangle \cdot\langle\mid \varnothing\rangle} \\
& =M(-q)^{2 \frac{\left(t_{1}+t_{2}\right)^{2}}{t_{1} t_{2}}}\left\langle\sigma_{1}(F)\right\rangle_{0} .
\end{aligned}
$$

To complete the calculation, we determine the series $\left\langle\sigma_{1}(F)\right\rangle_{0}$ via localization with respect to the $\mathbf{T}$-action defined in Section 2.2. Let the $\mathbf{T}$-equivariant lift of $F$ be 
specified by $\left[N_{0}\right]$. Then,

$$
\left\langle\sigma_{1}(F)\right\rangle_{0}=\left[\left.\left.\mathrm{W}_{\sigma_{1}(s)}(\varnothing, \varnothing, \varnothing)\right|_{s, t_{1}, t_{2}} \cdot \mathrm{W}(\varnothing, \varnothing, \varnothing)\right|_{-s, t_{1}, t_{2}}\right]_{s=0} .
$$

Lemma 18 The vertex measure $W_{\sigma_{1}(s)}(\lambda, \varnothing, \varnothing)$ with a descendent insertion is determined by

$$
\left.\mathrm{W}_{\sigma_{1}(s)}(\lambda, \varnothing, \varnothing)\right|_{s, t_{1}, t_{2}}=\left.s\left(-q \frac{d}{d q}+c\left(\lambda ; t_{1}, t_{2}\right)+\frac{|\lambda|}{2}\left(t_{1}+t_{2}\right)\right) \mathrm{W}(\lambda, \varnothing, \varnothing)\right|_{s, t_{1}, t_{2}} .
$$

Here, $c\left(\lambda ; t_{1}, t_{2}\right)$ is the sum of the $\left(t_{1}, t_{2}\right)$-contents of all squares in $\lambda$

$$
c\left(\lambda ; t_{1}, t_{2}\right)=\sum_{(i, j) \in \lambda}\left(i t_{1}+j t_{2}\right) .
$$

Viewing $\lambda$ as a Young diagram, the sum is over the interior corners of the squares the corners closest to the origin. For $|\lambda| \leq 1$, the total content $c\left(\lambda ; t_{1}, t_{2}\right)$ vanishes.

Proof Let $\mathbf{T}$ act of $\mathbb{C}^{3}$ with tangent weights $s, t_{1}, t_{2}$ at the origin. Let $\pi$ be a 3dimensional partition with a single infinite leg of cross-section $\lambda$ in the direction of the tangent weight $s$. Let $I_{\pi}$ denote the corresponding $\mathbf{T}$-fixed ideal. We first compute the restriction

$$
\left.\operatorname{ch}_{3}(\mathfrak{I})\right|_{\left[I_{\pi}\right] \times 0},
$$

where $\mathfrak{I}$ is the universal ideal sheaf.

Let $s_{1}, s_{2}, s_{3}$ be the associated weights of the $\mathbf{T}$-action on the coordinates $x_{1}, x_{2}, x_{3}$ of $\mathbb{C}^{3}$,

$$
s_{1}=-s, \quad s_{2}=-t_{1}, s_{3}=-t_{2} .
$$

Consider a graded free resolution of $I_{\pi}$ of length 3 ,

$$
0 \rightarrow \bigoplus_{k} x^{c_{k}} A \rightarrow \bigoplus_{j} x^{b_{j}} A \rightarrow \bigoplus_{i} x^{a_{i}} A \rightarrow I_{\pi} \rightarrow 0,
$$

where $A=\mathbb{C}\left[x_{1}, x_{2}, x_{3}\right]$ is the coordinate ring and

$$
a_{i}, b_{j}, c_{k} \in \mathbb{Z}^{3}
$$

denote the degrees of the generators in each step.

Computing the Chern character via the resolution, we find

$$
\left.\operatorname{ch}_{3}(\mathfrak{I})\right|_{I_{\pi} \times 0}=\frac{1}{3 !}\left(\sum_{i}\left(s, a_{i}\right)^{3}-\sum_{j}\left(s, b_{j}\right)^{3}+\sum_{k}\left(s, c_{k}\right)^{3}\right) .
$$

Here, $s=\left(s_{1}, s_{2}, s_{3}\right)$, and $(s, v)$ denotes the standard inner product of $s$ and $v$ in $\mathbb{Z}^{3}$. 
By calculating the trace of the $\mathbf{T}$-representation defined by $A / I_{\pi}$, we obtain a second relation:

$$
\begin{aligned}
& 1-\sum_{i} e^{\left(s, a_{i}\right)}+\sum_{j} e^{\left(s, b_{j}\right)}-\sum_{k} e^{\left(s, c_{k}\right)}= \\
& \quad\left(1-e^{s_{2}}\right)\left(1-e^{s_{3}}\right) \sum_{(i, j) \in \lambda} e^{i s_{2}+j s_{3}}+\left(1-e^{s_{1}}\right)\left(1-e^{s_{2}}\right)\left(1-e^{s_{3}}\right) \sum_{p \in \pi^{\prime}} e^{(s, p)}
\end{aligned}
$$

where $\pi^{\prime}$ denotes $\pi$ minus the infinite leg. In particular, the renormalized volume $|\pi|$ is the number of squares in $\pi^{\prime}$.

Extracting the cubic term in (33), we find

$$
\begin{aligned}
\left.\operatorname{ch}_{3}(\mathfrak{I})\right|_{I_{\pi} \times 0} & =-s_{2} s_{3}\left(c\left(\lambda ; s_{2}, s_{3}\right)+\frac{|\lambda|}{2}\left(s_{2}+s_{3}\right)\right)+s_{1} s_{2} s_{3}|\pi| \\
& =t_{1} t_{2}\left(-s|\pi|+c\left(\lambda ; t_{1}, t_{2}\right)+\frac{|\lambda|}{2}\left(t_{1}+t_{2}\right)\right) .
\end{aligned}
$$

When applied to the computation of $\left.\mathrm{W}_{\sigma_{1}(s)}(\lambda, \varnothing, \varnothing)\right|_{s, t_{1}, t_{2}}$, the argument of the descendent $\sigma_{1}(s)$ and the equivariant push-forward together remove the prefactor $t_{1} t_{2}$. $\square$

By evaluation (6) of $\mathrm{W}(\varnothing, \varnothing, \varnothing)$ and Lemma 18 ,

$$
\mathrm{W}_{\sigma_{1}(s)}(\varnothing, \varnothing, \varnothing)=\left.\frac{\left(t_{1}+t_{2}\right)\left(t_{1}+s\right)\left(t_{2}+s\right)}{t_{1} t_{2}} \Phi(q) \mathrm{W}(\varnothing, \varnothing, \varnothing)\right|_{s, t_{1}, t_{2}} .
$$

After multiplying all the factors,

$$
\left\langle\varnothing\left|\sigma_{1}(F)\right| \varnothing\right\rangle=\left(t_{1}+t_{2}\right) \Phi(q) .
$$

\subsection{Proofs of Lemma 16 and Proposition 17}

The proof is via $T$-localization on $\bar{N}$. We follow the notation of Section 4.6 for the $T$-fixed points of $\bar{N}_{\infty}$. Let

$$
0_{\uparrow}, 0_{\rightarrow} \in \bar{N}_{\infty}
$$

be the two new $T$-fixed points with normal $T$-weights

$$
t_{1}-t_{2},-t_{2}, \text { and } t_{2}-t_{1},-t_{1}
$$

respectively. 
For $\ell(\mu)=\ell(v)$, consider the $T$-equivariant localization formula for the integral

$$
\begin{aligned}
\left\langle\mu\left|\sigma_{1}(F)\right| v([0])\right\rangle^{\bar{N}}= & \left\langle\mu\left|\sigma_{1}(F)\right| v([0])\right\rangle \\
& +\left.q^{d}\langle\mu \mid v([0])\rangle_{d, d} \cdot\left\langle\varnothing\left|\sigma_{1}(F)\right| \varnothing\right\rangle\right|_{t_{1}-t_{2},-t_{2}} \\
& +\left.q^{d}\langle\mu \mid v([0])\rangle_{d, d} \cdot\left\langle\varnothing\left|\sigma_{1}(F)\right| \varnothing\right\rangle\right|_{t_{2}-t_{1},-t_{1}} .
\end{aligned}
$$

After evaluation and rearrangement,

$$
\begin{aligned}
\left\langle\mu\left|\sigma_{1}(F)\right| v([0])\right\rangle=\left\langle\mu\left|\sigma_{1}(F)\right| v[(0)]\right\rangle^{\bar{N}} & \\
& +\frac{(-1)^{d-\ell(\mu)} \delta_{\mu, v}}{\mathfrak{z}(\mu)}\left(t_{1}+t_{2}\right) q^{d} \Phi(q)
\end{aligned}
$$

We find $\left\langle\mu\left|\sigma_{1}(F)\right| v[(0)]\right\rangle$ is a linear function of $t_{1}$ and $t_{2}$. By symmetry, the integral must be a function of $t_{1}+t_{2}$.

We draw two conclusions. First, in the diagonal case $\mu=v$, the form (31) is proven. Second, $\left\langle\mu\left|\sigma_{1}(F)\right| v[(0)]\right\rangle$ is determined by restriction to $t_{2}=0$.

If $\ell(v)=1$, the Lemma and Proposition are empty. Let $\ell(v) \geq 2$. We proceed by induction on $\ell(v)$.

The strategy of the proof is to compare two $T$-equivariant integrals:

$$
m\left(v_{1}\right)\left\langle\mu\left|\sigma_{1}(F)\right| v[(0)]\right\rangle_{n}^{\bar{N}}
$$

and

$$
\left\langle\mu\left|\sigma_{1}(F)\right| v_{1}\left(\left[0_{\uparrow}\right]\right), v_{2}[(0)], \ldots,\left.v_{\ell(v)}([0])\right|_{n} ^{\bar{N}},\right.
$$

where $m\left(v_{1}\right)$ is the multiplicity of the part $v_{1}$ in $v$. Both integrals evaluate to linear functions of $t_{1}$ and $t_{2}$. The answers are not equal - the integrands are different $T$-equivariant classes. Consider the 1 -dimensional subtorus

$$
T_{1} \subset T
$$

determined by the first factor. The points

$$
0,0 \uparrow \in \bar{N}_{\infty}
$$

define equivalent $T_{1}$-equivariant classes on $\bar{N}$. Hence, the two integrals above are equal after the restriction $t_{2}=0$.

Consider the $T$-equivariant localization formula for the second integral. The formula immediately yields 0 unless there is a part $\mu_{1}$ of $\mu$ equal to $v_{1}$. Let $\mu^{*}$ and $v^{*}$ denote 
the subpartitions obtained by removing the first parts of $\mu$ and $\nu$. Then,

$$
\begin{gathered}
\left\langle\mu\left|\sigma_{1}(F)\right| v_{1}\left(\left[0_{\uparrow}\right]\right),\left.v^{*}([0])\right|^{\bar{N}}=q^{\mu_{1}} \frac{(-1)^{\mu_{1}-1}}{\mu_{1}}\left\langle\mu^{*}\left|\sigma_{1}(F)\right| v^{*}([0])\right\rangle\right. \\
+\left.q^{\left|\mu^{*}\right|} \frac{(-1)^{\left|\mu^{*}\right|-\ell\left(\mu^{*}\right)} \delta_{\mu^{*}, v^{*}}}{\mathfrak{z}\left(\mu^{*}\right)}\left\langle\mu_{1}\left|\sigma_{1}(F)\right| v_{1}([0])\right\rangle\right|_{t_{1}-t_{2},-t_{2}} \\
+q^{d} \frac{(-1)^{\mu_{1}-1}}{\mu_{1}} \frac{(-1)^{\left|\mu^{*}\right|-\ell\left(\mu^{*}\right)} \delta_{\mu^{*}, v^{*}}}{\mathfrak{z}\left(\mu^{*}\right)}\left(-2 t_{1}+t_{2}\right) \Phi(q)
\end{gathered}
$$

Comparing the $t_{2}=0$ restriction with (35) and using induction, we find

$$
\left\langle\mu\left|\sigma_{1}(F)\right| v[(0)]\right\rangle=0
$$

unless $\mu=v$ proving Lemma 16 .

We now assume $\mu=v$. Combining all the equations yields the following additivity relation:

$$
\frac{\left\langle\mu\left|\sigma_{1}(F)\right| \mu\right\rangle}{\langle\mu \mid \mu\rangle_{|\mu|,|\mu|}}=q^{\mu_{1}} \frac{\left\langle\mu^{*}\left|\sigma_{1}(F)\right| \mu^{*}\right\rangle}{\langle\mu \mid \mu\rangle_{\left|\mu^{*}\right|,\left|\mu^{*}\right|}}+q^{\left|\mu^{*}\right|} \frac{\left\langle\mu_{1}\left|\sigma_{1}(F)\right| \mu_{1}\right\rangle}{\left\langle\mu_{1} \mid \mu_{1}\right\rangle_{\mu_{1}, \mu_{1}}}-\left(t_{1}+t_{2}\right) q^{d} \Phi(q) .
$$

The induction step is complete and Proposition 17 is proven.

\section{Degree 1}

\subsection{Vertex measure}

The following formula for the degree 1 vertex measure was stated (without proof) in Maulik et al [25].

Proposition 19 At a 3-fold fixed point with tangent weights $s_{1}, s_{2}$, and $s_{3}$, the degree 1 vertex measure is

$$
\mathrm{W}(1, \varnothing, \varnothing)=(1+q)^{\frac{s_{2}+s_{3}}{s_{1}}} M(-q)^{-\frac{\left(s_{1}+s_{2}\right)\left(s_{1}+s_{3}\right)\left(s_{2}+s_{3}\right)}{s_{1} s_{2} s_{3}}},
$$

where the unique degree 1 leg extends in the $s_{1}$ direction.

As an easy application of Proposition 19, we calculate the series

$$
\langle\rangle_{1}=\sum_{n \geq 1} q^{n}\langle\rangle_{n, 1}
$$


via T-localization:

$$
\begin{aligned}
\langle\rangle_{1} & =\left[\left.\left.\mathrm{W}(1, \varnothing, \varnothing)\right|_{s, t_{1}, t_{2}} \cdot \frac{q}{t_{1} t_{2}} \cdot \mathrm{W}(1, \varnothing, \varnothing)\right|_{-s, t_{1}, t_{2}}\right]_{s=0} \\
& =\frac{q}{t_{1} t_{2}} M(-q)^{-2 \frac{\left(t_{1}+t_{2}\right)^{2}}{t_{1} t_{2}}} .
\end{aligned}
$$

Empty Donaldson-Thomas brackets \langle\rangle denote the integrand 1,

$$
\langle\rangle_{1}=\langle 1\rangle_{1} \text {. }
$$

\subsection{Proof of Proposition 19}

The Proposition is proven by using two geometric constraints for the vertex measure $\mathrm{W}(1,0,0)$.

The effective curve classes of $\mathbf{P}^{1} \times \mathbf{P}^{2}$ are generated by $\beta_{1}$ and $\beta_{2}$ where

$$
\beta_{1}=\left[\mathbf{P}^{1} \times 0\right], \quad \beta_{2}=\left[0 \times \mathbf{P}^{1}\right] .
$$

We will calculate Donaldson-Thomas series associated to these two classes.

Consider first $\beta_{1}$. The virtual dimension of $I_{n}\left(\mathbf{P}^{1} \times \mathbf{P}^{2}, \beta_{1}\right)$ is 2 . There is a HilbertChow morphism

$$
\epsilon: I_{n}\left(\mathbf{P}^{1} \times \mathbf{P}^{2}, \beta_{1}\right) \rightarrow \mathbf{P}^{2}
$$

which specifies the location of the line over the second factor of $\mathbf{P}^{1} \times \mathbf{P}^{2}$. We will compute the Donaldson-Thomas series

$$
\frac{\left\langle\left.\epsilon^{*}(P)\right|_{\beta_{1}} ^{\mathbf{P}^{1} \times \mathbf{P}^{2}}\right.}{\langle\rangle_{0}^{\mathbf{P}^{1} \times \mathbf{P}^{2}}}=\frac{\sum_{n \geq 1} q^{n}\left\langle\left.\epsilon^{*}(P)\right|_{n, \beta_{1}} ^{\mathbf{P}^{1} \times \mathbf{P}^{2}}\right.}{\sum_{n \geq 0} q^{n}\langle\rangle_{n, 0}^{\mathbf{P}^{1} \times \mathbf{P}^{2}}}
$$

where $P$ is the class of a point in $\mathbf{P}^{2}$. The integrand in the numerator of (36) has dimension 2, so the integrals are well-defined.

We will calculate (36) by equivariant localization. Let the 1 -dimensional torus $S$ act on $\mathbf{P}^{1}$ with fixed points $0, \infty \in \mathbf{P}^{1}$ and tangent weights $s,-s$. Let the 2-dimensional torus $T$ act on $\mathbf{P}^{2}$ with fixed points $p, p^{\prime}, p^{\prime \prime}$. Let $t_{1}, t_{2}$ be the tangent $T$-weights at $p$. Localization of (36) by the action of the 3-dimensional torus,

$$
\mathbf{T}=S \times T,
$$

yields

$$
\frac{\left\langle\left.\epsilon^{*}(P)\right|_{\beta_{1}} ^{\mathbf{P}^{1} \times \mathbf{P}^{2}}\right.}{\langle\rangle_{0}^{\mathbf{P}^{1} \times \mathbf{P}^{2}}}=\left.\left.t_{1} t_{2} \frac{\mathrm{W}(1, \varnothing, \varnothing)}{\mathrm{W}(\varnothing, \varnothing, \varnothing)}\right|_{s, t_{1}, t_{2}} \cdot \frac{q}{t_{1} t_{2}} \cdot \frac{\mathrm{W}(1, \varnothing, \varnothing)}{\mathrm{W}(\varnothing, \varnothing, \varnothing)}\right|_{-s, t_{1}, t_{2}} .
$$


Here, the $\mathbf{T}$-equivariant lift of $P$ is specified by $P=[p]$. We conclude

$$
\left.\left.\mathrm{W}^{\prime}(1, \varnothing, \varnothing)\right|_{s, t_{1}, t_{2}} \mathrm{~W}^{\prime}(1, \varnothing, \varnothing)\right|_{-s, t_{1}, t_{2}} \in \mathbb{Q} \llbracket q \rrbracket,
$$

where

$$
\mathrm{W}^{\prime}(1, \varnothing, \varnothing)=\frac{\mathrm{W}(1, \varnothing, \varnothing)}{\mathrm{W}(\varnothing, \varnothing, \varnothing)}
$$

Next, consider the class $\beta_{2}$. The virtual dimension of $I_{n}\left(\mathbf{P}^{2} \times \mathbf{P}^{1}, \beta_{1}\right)$ is 3 . There is a Hilbert-Chow morphism

$$
\epsilon: I_{n}\left(\mathbf{P}^{1} \times \mathbf{P}^{2}, \beta_{2}\right) \rightarrow \mathbf{P}^{1} \times\left(\mathbf{P}^{2}\right)^{\vee}
$$

which specifies the line component. We will compute the Donaldson-Thomas series

$$
\frac{\left\langle\left.\epsilon^{*}(Q)\right|_{\beta_{2}} ^{\mathbf{P}^{1} \times \mathbf{P}^{2}}\right.}{\langle\rangle_{0}^{\mathbf{P}^{1} \times \mathbf{P}^{2}}}=\frac{\sum_{n \geq 1} q^{n}\left\langle\left.\epsilon^{*}(Q)\right|_{n, \beta_{2}} ^{\mathbf{P}^{1} \times \mathbf{P}^{2}}\right.}{\sum_{n \geq 0} q^{n}\langle\rangle_{n, 0}^{\mathbf{P}^{1} \times \mathbf{P}^{2}}}
$$

where $Q$ is the class of a point in $\mathbf{P}^{1} \times\left(\mathbf{P}^{2}\right)^{\vee}$. The integrand in the numerator of (36) has dimension 3 , so the integrals are well-defined.

The $\mathbf{T}$-equivariant localization formula yields the following evaluation of the series (38):

$$
\frac{\left\langle\left.\epsilon^{*}(Q)\right|_{\beta_{2}} ^{\mathbf{P}^{1} \times \mathbf{P}^{2}}\right.}{\langle\rangle_{0}^{\mathbf{P}^{1} \times \mathbf{P}^{2}}}=\left.\left.s t_{2}\left(t_{2}-t_{1}\right) \frac{\mathrm{W}(1, \varnothing, \varnothing)}{\mathrm{W}(\varnothing, \varnothing, \varnothing)}\right|_{t_{1}, s, t_{2}} \cdot \frac{q}{s t_{2}\left(t_{2}-t_{1}\right)} \cdot \frac{\mathrm{W}(1, \varnothing, \varnothing)}{\mathrm{W}(\varnothing, \varnothing, \varnothing)}\right|_{-t_{1}, s, t_{2}-t_{1}} .
$$

The $\mathbf{T}$-equivariant lift of $Q$ is specified by the line over $0 \in \mathbf{P}^{1}$ connecting $T$-fixed points $p, p^{\prime} \in \mathbf{P}^{2}$ where the tangent $T$-weights at $p^{\prime}$ are $-t_{1}, t_{2}-t_{1}$. We conclude

$$
\left.\left.\mathrm{W}^{\prime}(1, \varnothing, \varnothing)\right|_{t_{1}, s, t_{2}} \mathrm{~W}^{\prime}(1, \varnothing, \varnothing)\right|_{-t_{1}, s, t_{2}-t_{1}} \in \mathbb{Q} \llbracket q \rrbracket .
$$

After renaming the variables, we obtain

$$
\left.\left.\mathrm{W}^{\prime}(1, \varnothing, \varnothing)\right|_{s, t_{1}, t_{2}} \mathrm{~W}^{\prime}(1, \varnothing, \varnothing)\right|_{-s, t_{1}, t_{2}-s} \in \mathbb{Q} \llbracket q \rrbracket,
$$

and, by symmetry,

$$
\left.\left.\mathrm{W}^{\prime}(1, \varnothing, \varnothing)\right|_{s, t_{1}, t_{2}} \mathrm{~W}^{\prime}(1, \varnothing, \varnothing)\right|_{-s, t_{1}-s, t_{2}} \in \mathbb{Q} \llbracket q \rrbracket,
$$

By definition of the equivariant vertex measure,

$$
\left.\mathrm{W}(1, \varnothing, \varnothing)\right|_{s, t_{1}, t_{2}} \in \mathbb{Q}\left(s, t_{1}, t_{2}\right) \llbracket q \rrbracket .
$$

The $q^{0}$ coefficient is 1 . By Lemma 6 , the coefficient of $q^{n}$ is divisible by $t_{1}+t_{2}$ for $n>0$. 
By repeated applications of the logarithms of equations (37), (39), and (40), we find

$$
\left.\log \left(\mathrm{W}^{\prime}(1, \varnothing, \varnothing)\right)\right|_{s, t_{1}, t_{2}}+\left.\log \left(\mathrm{W}^{\prime}(1, \varnothing, \varnothing)\right)\right|_{-s, t_{1}-i s, t_{2}-j s} \in \mathbb{Q} \llbracket q \rrbracket
$$

for all non-negative integers $i$ and $j$. The coefficients

$$
\left.\log \left(\mathrm{W}^{\prime}(1, \varnothing, \varnothing)\right)\right|_{s, t_{1}, t_{2}}=\sum_{n \geq 1} f_{n}\left(s, t_{1}, t_{2}\right) q^{n}
$$

must therefore satisfy

$$
f_{n}\left(s, t_{1}, t_{2}\right)+f_{n}\left(-s, t_{1}-x s, t_{2}-y s\right)=g_{n}(x, y)
$$

for variables $x$ and $y$. Differentiation with respect to $x$ yields

$$
-s \frac{\partial f_{n}}{\partial t_{1}}\left(-s, t_{1}, t_{2}\right)=\frac{\partial g_{n}}{\partial x}(0,0)
$$

Similarly

$$
-s \frac{\partial f_{n}}{\partial t_{2}}\left(-s, t_{1}, t_{2}\right)=\frac{\partial g_{n}}{\partial y}(0,0) .
$$

Hence, by integration and symmetry,

$$
f_{n}\left(s, t_{1}, t_{2}\right)=\gamma_{n} \frac{t_{1}+t_{2}}{s},
$$

where $\gamma_{n} \in \mathbb{Q}$. Since $f_{n}$ must be divisible by $t_{1}+t_{2}$, the constant of integration vanishes.

After specializing to Calabi-Yau weights $s+t_{1}+t_{2}=0$, we find

$$
f_{n}=-\gamma_{n} .
$$

However, after Calabi-Yau specialization, the equivariant vertex measure takes the simple form

$$
w(\pi)=(-1)^{|\pi|}
$$

for 3-dimensional partitions $\pi$, see Maulik et al [24].

Lemma $\left.20 \log \left(\mathrm{W}^{\prime}(1, \varnothing, \varnothing)\right)\right|_{s+t_{1}+t_{2}=0}=-\log (1+q)$.

The Lemma is a special case of the vertex evaluation required for the calculation of the level $(-1,0)$ cap (see Okounkov-Reshetikhin [32]), see Section 10.7 for a detailed discussion.

Lemma 20 determines the constants $\gamma_{n}$ and completes the proof of Proposition 19. 


\subsection{Descendent calculation}

We calculate the series $\left\langle(1)\left|\sigma_{1}(F)\right|(1)\right\rangle$. By the degeneration formula,

$$
\begin{aligned}
\left\langle(1)\left|\sigma_{1}(F)\right|(1)\right\rangle & =\frac{q^{2}}{\left(t_{1} t_{2}\right)^{2}} \frac{\left\langle\sigma_{1}(F)\right\rangle_{1}}{\langle(1) \mid\rangle \cdot\langle\mid(1)\rangle} \\
& =\frac{q}{t_{1} t_{2}} \frac{\left\langle\sigma_{1}(F)\right\rangle_{1}}{\langle\rangle_{1}} \\
& =M(-q)^{2 \frac{\left(t_{1}+t_{2}\right)^{2}}{t_{1} t_{2}}}\left\langle\sigma_{1}(F)\right\rangle_{1} .
\end{aligned}
$$

To complete the calculation, we determine the series $\left\langle\sigma_{1}(F)\right\rangle_{1}$ via localization with respect to the $\mathbf{T}$-action. Let the $\mathbf{T}$-equivariant lift of $F$ be specified by $\left[N_{0}\right]$. Then,

$$
\left\langle\sigma_{1}(F)\right\rangle_{1}=\left[\left.\left.\mathrm{W}_{\sigma_{1}(s)}(1, \varnothing, \varnothing)\right|_{s, t_{1}, t_{2}} \cdot \frac{q}{t_{1} t_{2}} \cdot \mathrm{W}(1, \varnothing, \varnothing)\right|_{-s, t_{1}, t_{2}}\right]_{s=0} .
$$

By Proposition 19 and Lemma 18,

$$
\begin{aligned}
\mathrm{W}_{\sigma_{1}(s)}(1, \varnothing, \varnothing)=( & \left.t_{1}+t_{2}\right)\left(\frac{-q}{1+q}+\frac{1}{2}\right) \mathrm{W}(1, \varnothing, \varnothing)_{s, t_{1}, t_{2}} \\
& +\left.\frac{\left(t_{1}+t_{2}\right)\left(t_{1}+s\right)\left(t_{2}+s\right)}{t_{1} t_{2}} \Phi(q) \mathrm{W}(1, \varnothing, \varnothing)\right|_{s, t_{1}, t_{2}} .
\end{aligned}
$$

After multiplying all the factors,

$$
\frac{\left\langle(1)\left|\sigma_{1}(F)\right|(1)\right\rangle}{\langle(1) \mid(1)\rangle_{1,1}}=\frac{\left(t_{1}+t_{2}\right)}{2} q \frac{1-q}{1+q}+\left(t_{1}+t_{2}\right) q \Phi(q) .
$$

\subsection{Cap}

We calculate the partition function $\operatorname{DT}(0 \mid 0,0)_{\lambda}$ corresponding to the cap in the TQFT formalism.

Lemma 21 The invariants of the level $(0,0)$ cap are given by

$$
\operatorname{DT}(0 \mid 0,0)_{\lambda}= \begin{cases}\frac{1}{d !\left(t_{1} t_{2}\right)^{d}} & \text { if } \lambda=\left(1^{d}\right) \\ 0 & \text { if } \lambda \neq\left(1^{d}\right) .\end{cases}
$$

Proof If $\lambda \neq\left(1^{d}\right)$, then $\ell(\lambda)<d$. By equation (18),

$$
\langle\mid \lambda([0])\rangle=0
$$


if $\ell(\lambda)<d$. Since

$$
\operatorname{DT}(0 \mid 0,0)_{\lambda}=q^{-d} \frac{\langle\mid \lambda\rangle}{\langle\mid \varnothing\rangle}=\frac{q^{-d}}{\left(t_{1} t_{2}\right)^{\ell(\lambda)}} \frac{\langle\mid \lambda([0])\rangle}{\langle\mid \varnothing\rangle},
$$

the cap DT $(0 \mid 0,0)_{\lambda}$ vanishes if $\lambda \neq\left(1^{d}\right)$.

If $\lambda=\left(1^{d}\right)$, then the Donaldson-Thomas invariant for the compactified geometry

$$
\langle\mid \lambda([0])\rangle^{\overline{(0,0)}}
$$

is a constant independent of the equivariant parameters $t_{1}$ and $t_{2}$. By specializing the partition weights to different $T$-fixed points and localizing, we obtain

$$
\frac{\langle\mid \lambda([0])\rangle}{\langle\mid \varnothing\rangle}=\frac{1}{d !}\left(\frac{\langle\mid 1([0])\rangle}{\langle\mid \varnothing\rangle}\right)^{d} \text {. }
$$

The degree 1 calculation,

$$
\frac{\langle\mid 1([0])\rangle}{\langle\mid \varnothing\rangle}=q
$$

completes the proof of the Lemma.

\section{The operator $M_{\sigma}$}

\subsection{Fock space formalism}

Let the 2-dimensional torus $T$ act on $\mathbb{C}^{2}$ by standard diagonal scaling. We review the Fock space description of the $T$-equivariant cohomology of the Hilbert scheme of points of $\mathbb{C}^{2}$, see Grojnowski [10] and Nakajima [28].

By definition, the Fock space $\mathcal{F}$ is freely generated over $\mathbb{Q}$ by commuting creation operators $\alpha_{-k}, k \in \mathbb{Z}_{>0}$, acting on the vacuum vector $v_{\varnothing}$. The annihilation operators $\alpha_{k}, k \in \mathbb{Z}_{>0}$, kill the vacuum

$$
\alpha_{k} \cdot v_{\varnothing}=0, \quad k>0,
$$

and satisfy the commutation relations

$$
\left[\alpha_{k}, \alpha_{l}\right]=k \delta_{k+l} .
$$

A natural basis of $\mathcal{F}$ is given by the vectors

$$
|\mu\rangle=\frac{1}{\mathfrak{z}(\mu)} \prod \alpha_{-\mu_{i}} v_{\varnothing} .
$$


indexed by partitions $\mu$. Here,

$$
\mathfrak{z}(\mu)=|\operatorname{Aut}(\mu)| \prod \mu_{i}
$$

is the usual normalization factor.

The Nakajima basis defines a canonical isomorphism,

$$
\mathcal{F} \otimes_{\mathbb{Q}} \mathbb{Q}\left[t_{1}, t_{2}\right] \cong \bigoplus_{d \geq 0} A_{T}^{*}\left(\operatorname{Hilb}\left(\mathbb{C}^{2}, d\right), \mathbb{Q}\right)
$$

The Nakajima basis element corresponding to $|\mu\rangle$ is

$$
\frac{1}{\Pi_{i} \mu_{i}}\left[V_{\mu}\right]
$$

where $\left[V_{\mu}\right]$ is (the cohomological dual of) the class of the subvariety of $\operatorname{Hilb}\left(\mathbb{C}^{2},|\mu|\right)$ with generic element given by a union of schemes of lengths

$$
\mu_{1}, \ldots, \mu_{\ell(\mu)}
$$

supported at $\ell(\mu)$ distinct points of $\mathbb{C}^{2}$. The vacuum vector $v_{\varnothing}$ corresponds to the unit in $A_{T}^{*}\left(\mathrm{Hilb}_{0}, \mathbb{Q}\right)$.

The standard inner product on the $T$-cohomology induces the following nonstandard inner product on Fock space after an extension of scalars:

$$
\langle\mu \mid \nu\rangle_{\mathcal{F}}=\frac{1}{\left(t_{1} t_{2}\right)^{\ell(\mu)}} \frac{(-1)^{|\mu|-\ell(\mu)}}{\mathfrak{z}(\mu)} \delta_{\mu \nu} .
$$

With respect to the inner product,

$$
\left(\alpha_{k}\right)^{*}=(-1)^{k-1}\left(t_{1} t_{2}\right)^{\operatorname{sgn}(k)} \alpha_{-k} .
$$

If there is no ambiguity, the subscript $\mathcal{F}$ will be omitted from the bracket (43).

\subsection{The class $D$}

Let $\mathcal{O} / \mathcal{I}$ be the rank $d$ tautological bundle on $\operatorname{Hilb}\left(\mathbb{C}^{2}, d\right)$, and let

$$
D=c_{1}(\mathcal{O} / \mathcal{I}) .
$$

A straightforward calculation shows

$$
D=-\left|2,1^{d-2}\right\rangle .
$$


The classical multiplication of $D$ on the Fock space $\mathcal{F}$ is given by the following operator:

$$
-\left(t_{1}+t_{2}\right) \sum_{k>0} \frac{k-1}{2} \alpha_{-k} \alpha_{k}+\frac{1}{2} \sum_{k, l>0}\left[t_{1} t_{2} \alpha_{k+l} \alpha_{-k} \alpha_{-l}-\alpha_{-k-l} \alpha_{k} \alpha_{l}\right],
$$

see Lehn [14], Lehn-Sorger [15], Li et al [20] and Okounkov-Pandharipande [31].

The first summand of (45) contains a term proportional to the energy operator,

$$
|\cdot|=\sum_{k>0} \alpha_{-k} \alpha_{k}
$$

The energy operator acts diagonally on Fock space with eigenvalue $|\mu|$ on the vector $|\mu\rangle$.

\subsection{Operators}

The following operator on Fock space plays a central role in the paper:

$$
\begin{aligned}
\mathrm{M}\left(q, t_{1}, t_{2}\right)=\left(t_{1}+t_{2}\right) \sum_{k>0} \frac{k}{2} \frac{(-q)^{k}+1}{(-q)^{k}-1} \alpha_{-k} \alpha_{k}+ \\
\quad \frac{1}{2} \sum_{k, l>0}\left[t_{1} t_{2} \alpha_{k+l} \alpha_{-k} \alpha_{-l}-\alpha_{-k-l} \alpha_{k} \alpha_{l}\right] .
\end{aligned}
$$

The $q$-dependence of $\mathrm{M}$ is only in the first sum in (46) which acts diagonally in the basis (42). The two terms in the second sum in (46) are known respectively as the splitting and joining terms. The operator $M$ is self-adjoint

$$
\mathrm{M}^{*}=\mathrm{M}
$$

with respect to (44).

Let the operator $M_{\sigma}$ on Fock space be defined by matrix elements

$$
\begin{aligned}
\left\langle\mu\left|\mathrm{M}_{\sigma}\right| v\right\rangle_{\mathcal{F}} & =q^{-d}\left\langle\mu\left|-\sigma_{1}(F)\right| v\right\rangle \\
& =\sum_{n \geq d} q^{n-d}\left\langle\mu\left|-\sigma_{1}(F)\right| v\right\rangle_{n, d}
\end{aligned}
$$

for partitions satisfying

$$
|\mu|=|v|=d .
$$

The matrix elements of $\mathrm{M}_{\sigma}$ are understood to vanish unless $|\mu|=|v|$. Define the operator

$$
\widetilde{\mathrm{M}}_{\sigma}=\mathrm{M}-\left(t_{1}+t_{2}\right) \Phi(q) \cdot \mathrm{Id}
$$




\section{Proposition $22 \quad M_{\sigma}=\widetilde{M}_{\sigma}$}

We check here three initial compatibilities required for Proposition 22. Proposition 22 will be proven in Section 8 by relating Donaldson-Thomas integrals to Gromov-Witten invariants of the Hilbert scheme $\operatorname{Hilb}\left(\mathbb{C}^{2}, d\right)$.

First, Proposition 22 requires the negative of the $q$-shifted operator bracket,

$$
-q^{d}\left\langle\mu\left|\widetilde{\mathrm{M}}_{\sigma}\right| v\right\rangle_{\mathcal{F}},
$$

to satisfy the additivity property of Proposition 17 . The required additivity is easily checked.

Second, Proposition 22 is valid for $q=0$. Let $N$ be the trivial bundle with level $(0,0)$ splitting on $\mathbf{P}^{1}$. There is an isomorphism of moduli spaces

$$
I_{d}\left(N / N_{0} \cup N_{\infty}, d\right) \cong \operatorname{Hilb}\left(N_{z}, d\right)
$$

for any $z \in \mathbf{P}^{1}$. Under the isomorphism (48), the Donaldson-Thomas descendent class $-\sigma_{1}\left(\left[N_{z}\right]\right)$ determines an element of $A_{T}^{1}\left(\operatorname{Hilb}\left(N_{z}, d\right), \mathbb{Q}\right)$. By a Riemann-Roch calculation,

$$
-\sigma_{1}\left(N_{z}\right)=D-\frac{t_{1}+t_{2}}{2} d
$$

Proposition 22 correctly equates the $q=0$ evaluation $\widetilde{\mathrm{M}}_{\sigma}(0)$ of $\widetilde{\mathrm{M}}_{\sigma}$ with the classical multiplication (45) of $D$ shifted by $-\frac{t_{1}+t_{2}}{2} d$.

Third, Proposition 22 is valid in degrees 0 and 1 by the descendent evaluation (34),

$$
\left\langle\varnothing\left|\mathrm{M}_{\sigma}\right| \varnothing\right\rangle_{\mathcal{F}}=\left\langle\varnothing\left|-\sigma_{1}(F)\right| \varnothing\right\rangle=-\left(t_{1}+t_{2}\right) \Phi(q),
$$

and the descendent evaluation (41),

$$
\begin{aligned}
\frac{\left\langle(1)\left|\mathrm{M}_{\sigma}\right|(1)\right\rangle_{\mathcal{F}}}{\langle(1) \mid(1)\rangle_{\mathcal{F}}} & =q^{-1} \frac{\left\langle(1)\left|-\sigma_{1}(F)\right|(1)\right\rangle}{\langle(1) \mid(1)\rangle_{1,1}} \\
& =-\frac{\left(t_{1}+t_{2}\right)}{2} \frac{1-q}{1+q}-\left(t_{1}+t_{2}\right) \Phi(q) .
\end{aligned}
$$

\section{Proof of Proposition 22}

\subsection{Induction strategy}

Our proof of Proposition 22 closely follows the proof of Theorem 1 in OkounkovPandharipande [31] though several differences occur. Since the results and the geometry differ, the closeness of the proof is somewhat surprising. 
We proceed by induction on the degree $d$. If $d=0$ or 1 , Proposition 22 has already been proven by descendent calculations. Let $d>1$.

Next, we induct on the Euler number $n$. In the minimal case $n=d$, Proposition 22 recovers classical multiplication by $D$ on the Hilbert scheme of points. Let $n>d$.

The induction step relies upon the addition formula of Proposition 17. We will compute an invariant

$$
\left\langle\gamma_{1}\left|-\sigma_{1}(F)\right| \gamma_{2}\right\rangle_{n}
$$

for which the expansions of the classes

$$
\gamma_{1}, \gamma_{2} \in A_{T}^{2 d}\left(\operatorname{Hilb}\left(\mathbb{C}^{2}, d\right), \mathbb{Q}\right)
$$

in the Nakajima basis contain nontrivial multiples not divisible by $\left(t_{1}+t_{2}\right)$ of the class $|(d)\rangle$. By the addition rules, if

$$
\left\langle\gamma_{1}\left|-\sigma_{1}(F)\right| \gamma_{2}\right\rangle_{n}=\left\langle\gamma_{1}\left|\widetilde{M}_{\sigma}\right| \gamma_{2}\right\rangle_{\mathcal{F}, n-d},
$$

then Proposition 22 is proven for Euler number $n$.

Both sides of (49) are constant multiples of $t_{1}^{2 d}\left(t_{1}+t_{2}\right)$ modulo $\left(t_{1}+t_{2}\right)^{2}$. By (31),

$$
\left\langle(d)\left|-\sigma_{1}(F)\right|(d)\right\rangle_{n}=\frac{\gamma_{d, n}}{t_{1}^{2}}\left(t_{1}+t_{2}\right) \quad \bmod \left(t_{1}+t_{2}\right)^{2} .
$$

Hence, we need only verify the equality (49) modulo $\left(t_{1}+t_{2}\right)^{2}$.

\subsection{Induction step: I}

Let $d>1$ and let $n>d$. For the induction step, we will compute the invariant

$$
\left\langle\left[\mathcal{I}_{(d)}\right]\left|-\sigma_{1}(F)\right|\left[\mathcal{I}_{(d-1,1)}\right]\right\rangle_{n} .
$$

Here, $\mathcal{I}_{\lambda}$ denotes the monomial ideal corresponding to the partition $\lambda$, and $\left[\mathcal{I}_{\lambda}\right]$ denotes the $T$-equivariant class of the associated fixed point in $\operatorname{Hilb}\left(\mathbb{C}^{2},|\lambda|\right)$.

The $T$-fixed point $\left[\mathcal{I}_{\lambda}\right]$ corresponds to the Jack polynomial

$$
\mathrm{J}^{\lambda} \in \mathcal{F} \otimes \mathbb{Q}\left[t_{1}, t_{2}\right] .
$$

For $-t_{2} / t_{1}=1$, the Jack polynomials specialize to the Schur functions. Hence,

$$
J^{\lambda} \equiv \frac{(-1)^{|\lambda|}|\lambda| !}{\operatorname{dim} \lambda} \sum_{\mu} \chi_{\mu}^{\lambda} t_{1}^{|\lambda|+\ell(\mu)}|\mu\rangle \bmod t_{1}+t_{2},
$$

where $\operatorname{dim} \lambda$ is the dimension of the representation $\lambda$ of the symmetric group and $\chi_{\mu}^{\lambda}$ is the evaluation of the corresponding character on the conjugacy class $\mu$, see 
Macdonald [22]. In particular, the coefficient of $|d\rangle$ in the expansion of both $\mathrm{J}^{(d)}$ and $\mathrm{J}^{(d-1,1)}$ is nonzero.

\section{Lemma 23 We have}

$$
\begin{aligned}
&\left\langle\mathrm{J}^{(d)}\left|\widetilde{\mathrm{M}}_{\sigma}-\widetilde{\mathrm{M}}_{\sigma}(0)\right| \mathrm{J}^{(d-1,1)}\right\rangle_{\mathcal{F}} \equiv \\
& \quad(-1)^{d}\left(t_{1}+t_{2}\right) \frac{t_{1}^{2 d}(d !)^{2}}{d-1}\left(\frac{q}{1+q}+d \frac{(-q)^{d}}{1-(-q)^{d}}\right) \bmod \left(t_{1}+t_{2}\right)^{2}
\end{aligned}
$$

Here, the operator $\widetilde{M}_{\sigma}-\widetilde{M}_{\sigma}(0)$ is formed by the terms of positive degree in $q$ in the operator $\widetilde{M}_{\sigma}$.

The proof of Lemma 23 follows exactly the derivation of equation (31) of OkounkovPandharipande [31]. The differences between our operator $\widetilde{M}_{\sigma}$ and the operator $M_{D}$ of [31] yield constant functions orthogonal to the non-trivial character $\chi^{(d-1,1)}$.

\subsection{Localization}

8.3.1 Overview Our goal now is to reproduce the answer (51) by calculating the Donaldson-Thomas invariant

$$
\left\langle\left[\mathcal{I}_{(d)}\right]\left|-\sigma_{1}(F)\right|\left[\mathcal{I}_{(d-1,1)}\right]\right\rangle_{n}
$$

By the rubber calculus (29),

$$
\left\langle\left[\mathcal{I}_{(d)}\right]\left|-\sigma_{1}(F)\right|\left[\mathcal{I}_{(d-1,1)}\right]\right\rangle_{n}=(n-d)\left\langle\left[\mathcal{I}_{(d)}\right] \mid\left[\mathcal{I}_{(d-1,1)}\right]\right\rangle_{n}^{\sim}
$$

We calculate the right side of (52) by $T$-equivariant localization on the rubber moduli space

$$
R_{n, d}=I_{n}\left(R / R_{0} \cup R_{\infty}\right)^{\sim},
$$

see Section 4.8 .

Since the $T$-fixed locus of the moduli space $R_{n, d}$ is proper, the virtual localization formula of Graber-Pandharipande [9] may be applied. However, since $R_{n, d}$ contains positive dimensional families of $T$-invariant ideal sheaves, a straightforward application is difficult. Our strategy for computing (52) uses comparisons to integrals in the quantum cohomology of the Hilbert scheme of points $\operatorname{Hilb}\left(\mathbb{C}^{2}, d\right)$. 
8.3.2 Skewers and twistors Consider the rubber moduli space $R_{n, d}$ for $n>d$. Let

$$
[I] \in R_{n, d}^{T}
$$

be a $T$-fixed ideal sheaf. The ideal sheaf $I$ is defined on a rubber target fibered by $\mathbb{C}^{2}$ over a chain $C$ of rational curves. The diagram below gives an example of a subscheme associated to a $T$-fixed ideal sheaf on a reducible rubber target.

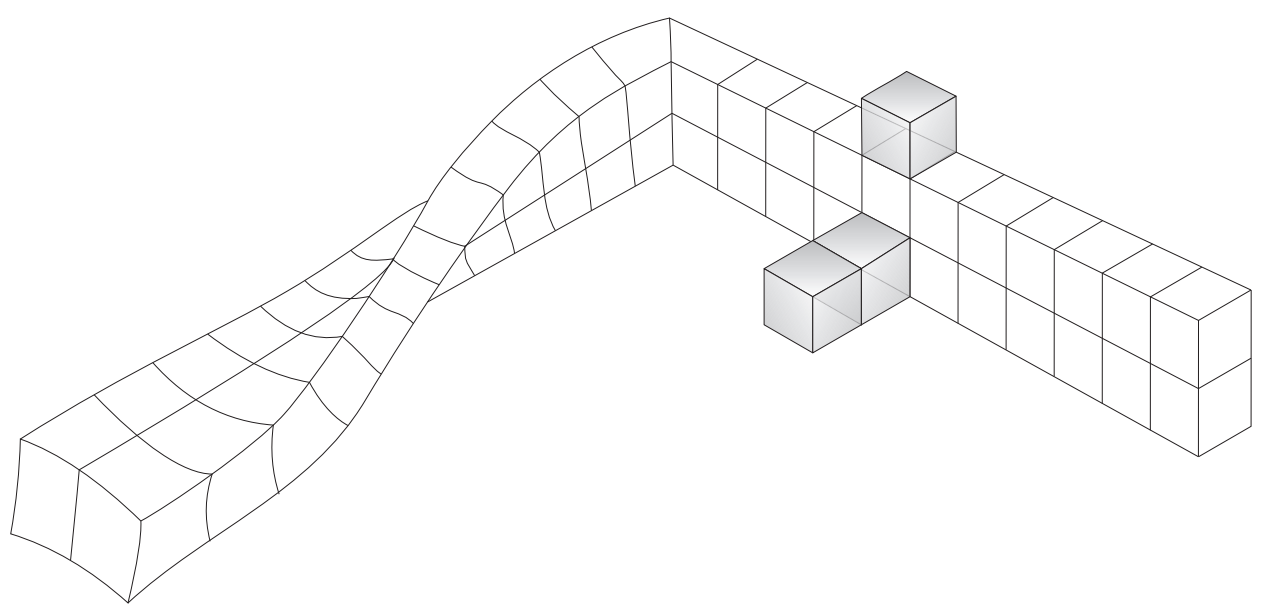

Let $P \subset C$ be a rational component. The restriction $I_{P}$ of $I$ to the component $\mathbb{C}^{2} \times P$ of the target rubber is either a skewer or a twistor:

(i) A skewer is determined by an element

$$
\left[I_{\mathrm{sk}}\right] \in I_{*}\left(\mathbb{C}^{2} \times \mathbf{P}^{1}, d\right)^{T}
$$

where the factor $\mathbf{P}^{1}$ is rigid. Certainly the skewer $I_{\mathrm{sk}}$ is $T$-fixed on the rubber $\mathbb{C}^{2} \times P$. The component on the right of the above diagram is a skewer.

(ii) A twistor is obtained from a $T$-fixed element

$$
\left[f_{\mathrm{tw}}\right] \in M_{0,\{0, \infty\}}\left(\operatorname{Hilb}\left(\left(\mathbb{C}^{2}, d\right), *\right)\right)^{T} .
$$

By pulling-back the universal ideal sheaf over the Hilbert scheme, $f_{\mathrm{tw}}$ determines a $T$-fixed ideal sheaf $I_{\mathrm{tw}}$ on the rubber $\mathbb{C}^{2} \times P$. The component on the left of the diagram is a twistor.

The degree of the map $f_{\mathrm{tw}}$ to the Hilbert scheme and the Euler number $n_{\mathrm{tw}}$ of $I_{\mathrm{tw}}$ are related by

$$
\operatorname{deg}\left(f_{\mathrm{tw}}\right)=n_{\mathrm{tw}}-d
$$

Elementary considerations show these disjoint constructions exhaust all $T$-fixed ideal sheaves on the rubber $\mathbb{C}^{2} \times P$. 
8.3.3 Comparison We will calculate (52) via a comparison result.

\section{Proposition 24 We have}

$$
\left\langle\left[\mathcal{I}_{(d)}\right] \mid\left[\mathcal{I}_{(d-1,1)}\right]\right\rangle_{n}^{\sim}=\left\langle\left[\mathcal{I}_{(d)}\right] \mid\left[\mathcal{I}_{(d-1,1)}\right]\right\rangle_{n-d}^{\operatorname{Hilb}\left(\mathbb{C}^{2}, d\right)} \quad \bmod \left(t_{1}+t_{2}\right)^{2}
$$

Proposition 24 is proven in Section 8.3.5. As a first step, a simpler comparison result is obtained here.

Consider the open set of stable maps,

$$
U_{n, d} \subset \bar{M}_{0,\{0, \infty\}}\left(\operatorname{Hilb}\left(\mathbb{C}^{2}, d\right), n-d\right)
$$

for which the domain is a chain of rational curves. The open set $U_{n, d}$ carries a $T-$ equivariant Gromov-Witten obstruction theory via restriction. By pulling-back the universal ideal sheaf over the Hilbert scheme (as in the twistor construction), we obtain an open immersion

$$
\text { ı: } U_{n, d} \subset R_{n, d} \text {. }
$$

Hence, $U_{n, d}$ also carries a $T$-equivariant Donaldson-Thomas theory.

Lemma 25 The $T$-equivariant Gromov-Witten and Donaldson-Thomas obstruction theories of $U_{n, d}$ are canonically isomorphic.

\section{Proof Let}

$$
\left[f: C \rightarrow \operatorname{Hilb}\left(\mathbb{C}^{2}, d\right)\right] \in U_{n, d}
$$

be a stable map, and let

$$
\iota([f])=[I] \in R_{n, d}
$$

be the associated ideal sheaf. Since both obstruction theories can be defined relative to the degenerations of the domain, we need only check the Gromov-Witten complex associated to the cohomology $H^{*}\left(C, f^{*} T_{\mathrm{Hilb}}\right)$ matches the shift of the complex associated to $\operatorname{Ext}_{0}(I, I)$. For a point $p \in C$, the tangent to the Hilbert scheme may be viewed as $\operatorname{Ext}_{0}^{1}\left(I_{p}, I_{p}\right)$. Moreover,

$$
\operatorname{Ext}_{0}^{0}\left(I_{p}, I_{p}\right)=\operatorname{Ext}_{0}^{2}\left(I_{p}, I_{p}\right)=0
$$

The required matching is then a straightforward application of the Leray spectral sequence. 
8.3.4 Tangent representations Let $[I] \in R_{n, d}^{T}$ be a $T$-fixed ideal sheaf on a rubber target fibered by $\mathbb{C}^{2}$ over a chain $C$ of rational curves.

The special points of $C$ consist of $0, \infty \in C$ and all the nodes. Over the special points of $s \in C$, the ideal $I$ must correspond to $T$-fixed points $\left[I_{s}\right] \in \operatorname{Hilb}\left(\mathbb{C}^{2}, d\right)$.

Let $P \subset C$ be a component containing the special points $s, s^{\prime}$ of $C$. Fractional $T-$ weight $w_{P, s}$ and $w_{P, s^{\prime}}$ are defined by the $T$-representation of the tangent space to $P$ at $s$ and $s^{\prime}$ :

(i) If $P \subset C$ corresponds to a skewer, then

$$
\left[I_{s}\right]=\left[I_{s^{\prime}}\right]
$$

and $w_{P, s}=w_{P, s^{\prime}}=0$.

(ii) If $P \subset C$ corresponds to a twistor, then

$$
\left[I_{s}\right] \neq\left[I_{s^{\prime}}\right]
$$

and $w_{P, s}, w_{P, s^{\prime}} \neq 0$ modulo $\left(t_{1}+t_{2}\right)$, see Okounkov-Pandharipande [31].

In fact, in the twistor case, if

$$
\left[I_{s}\right]=\left[\mathcal{I}_{\mu}\right], \quad\left[I_{s^{\prime}}\right]=\left[\mathcal{I}_{\nu}\right]
$$

then

$$
w_{P, s}=w_{\mu, \nu}^{n_{P}-d}=-w_{\nu, \mu}^{n_{P}-d}=-w_{P, s^{\prime}},
$$

where $n_{P}$ is the Euler characteristic associated to $I_{P}$ and $w_{\mu, \nu}^{n}$ is the universal function defined in [31, Lemma 4] viewed here as a $T$-weight.

8.3.5 Proof of Proposition 24 We index the $T$-equivariant localization contributions to the invariants

$$
\left\langle\left[\mathcal{I}_{\mu}\right] \mid\left[\mathcal{I}_{v}\right]\right\rangle_{n}^{\sim}
$$

by graphs following [31]. An oriented chain of Euler number $n$ is a graph $\Gamma_{\mu, \nu}=$ $\left(V, v_{1}, v_{2}, \rho, E, S, \delta\right)$,

(i) $V$ is a finite vertex set with distinguished elements $v_{1}$ and $v_{2}$,

(ii) $\rho: V \rightarrow \mathcal{P}(d)$,

(iii) $E$ is a finite edge set,

(iv) $S \subset V$,

(v) $\delta: E \cup S \rightarrow \mathbb{Z}_{>0}$ is an assignment,

satisfying the following conditions 
(a) $\Gamma$ is a connected chain with initial vertex $v_{1}$ and final vertex $v_{2}$,

(b) $\rho\left(v_{1}\right)=\mathcal{I}_{\mu}, \rho\left(v_{2}\right)=\mathcal{I}_{v}$,

(c) if $v^{\prime}, v^{\prime \prime} \in V$ are connected by an edge, then $\rho\left(v^{\prime}\right) \neq \rho\left(v^{\prime \prime}\right)$,

(d) if $v \in V \backslash S$ has edge valence 2 with neighbors $v^{\prime}, v^{\prime \prime}$, then

$$
w_{\rho(v), \rho\left(v^{\prime}\right)}^{\delta\left(e\left(v, v^{\prime}\right)\right)}+w_{\rho(v), \rho\left(v^{\prime \prime}\right)}^{\delta\left(e\left(v, v^{\prime \prime}\right)\right)} \neq 0 \quad \bmod t_{1}+t_{2},
$$

(e) $\sum_{e \in E} \delta(e)+\sum_{s \in S} \delta(s)=n+d(|E|+|S|-1)$.

Let $[I] \in R_{n, d}^{T}$ be $T$-fixed ideal sheaf on a rubber target fibered by $\mathbb{C}^{2}$ over a chain $C$ of rational curves. We associate an oriented chain,

$$
\Gamma_{I}=\left(V, v_{1}, v_{2}, \rho, E, S, \delta\right),
$$

of Euler number $n$ to $I$ by the following construction. The vertex set

$$
V=V_{1} \cup V_{2} \cup V_{3},
$$

is a union of three disjoint subsets:

(1) $V_{1}$ is the set of maximal connected subcurves of skewer components of the base $C$,

(2) $V_{2}$ is the set of nodes of $s \in C$ for which the incident components $P, P^{\prime} \subset C$ are twistors and satisfy the breaking condition

$$
w_{P, s}+w_{P^{\prime}, s} \neq 0 \quad \bmod t_{1}+t_{2},
$$

(3) $V_{3}$ is subset of the marking $0, \infty \in C$ which lie on twistors.

The markings $0, \infty \in C$ are associated to elements of the union $V_{1} \cup V_{3}$ - the markings determine $v_{1}$ and $v_{2}$. The function $\rho$ is obtained from $I$. Chains of unbroken twistors of $C$ link the vertices of $V$. The edge set $E$ is determined by such chains. The set $S$ equals $V_{1}$. The degree assignment $\delta$ is obtained from the Euler number of the restriction of $I$ to the associated components.

The oriented chain $\Gamma_{I}$ is easily seen to satisfy conditions (a)-(e). Condition (c) is a consequence of [31, Lemma 5]. The chain $\Gamma_{I}$ is invariant as [I] varies in a connected component of the $T$-fixed locus of $R_{n, d}^{T}$.

Let $G_{n}(\mu, v)$ denote the finite set of oriented chains trees $\Gamma_{\mu, \nu}$ of Euler number $n$. Let

$$
R_{\Gamma} \subset R_{n, d}^{T}
$$


denote the substack of $T$-fixed maps corresponding to the tree $\Gamma \in G_{n}(\mu, \nu)$. Let

$$
\left\langle\left[\mathcal{I}_{\mu}\right] \mid\left[\mathcal{I}_{v}\right]\right\rangle_{d}^{\Gamma \sim}
$$

denote the localization contribution of $R_{\Gamma}$. By the virtual localization formula (see Graber-Pandharipande [9]),

$$
\left\langle\left[\mathcal{I}_{\mu}\right] \mid\left[\mathcal{I}_{\nu}\right]\right\rangle_{d}^{\Gamma \sim}=\int_{\left[R_{\Gamma}\right]^{\mathrm{vir}}} \frac{\epsilon_{0}^{*}\left(\left[\mathcal{I}_{\mu}\right]\right) \cup \epsilon_{\infty}^{*}\left(\left[\mathcal{I}_{\nu}\right]\right)}{e\left(N^{\mathrm{vir}}\right)} .
$$

By decomposing the Donaldson-Thomas obstruction theory, we can express the integral (53) in terms of descendent skewer integrals corresponding to the vertices $S$ and descendent twistor integrals corresponding to the edges $E$.

The edge integrals are exactly equal to associated integrals in the Gromov-Witten theory of the Hilbert scheme Hilb $\left(\mathbb{C}^{2}, d\right)$ by Lemma 25 . Moreover, the Hilbert scheme integrals which arise for each edge compute a Gromov-Witten residue for the $T^{ \pm}$ action. Hence, the edge term contributions to (53) are divisible by $\left(t_{1}+t_{2}\right)$.

We prove the skewer integrals are also divisible by $\left(t_{1}+t_{2}\right)$ by localizing a rubber integral:

$$
\left\langle\left[\mathcal{I}_{\gamma}\right]\left|\psi_{0}^{a} \psi_{\infty}^{b}\right|\left[\mathcal{I}_{\gamma}\right]\right)_{n}^{\sim}=\left\langle\left[\mathcal{I}_{\gamma}\right]\left|\psi_{0}^{a} \psi_{\infty}^{b}\right|\left[\mathcal{I}_{\gamma}\right]\right\rangle^{\Gamma_{\gamma, \gamma}^{0} \sim}+\sum_{\Gamma \in G_{n}(\gamma, \gamma) \backslash\left\{\Gamma^{0}\right\}}\left\langle\left[\mathcal{I}_{\gamma}\right]\left|\psi_{0}^{a} \psi_{\infty}^{b}\right|\left[\mathcal{I}_{\gamma}\right]\right\rangle^{\Gamma \sim}
$$

Here, $\Gamma_{\gamma, \gamma}^{0}$ is the unique chain with a single skewer vertex. By Lemma 13, the rubber integral on the left is divisible by $\left(t_{1}+t_{2}\right)$. The $\Gamma^{0}$ contribution is the skewer integral of interest. The second summand on the right is expressed in terms of skewer integrals of lower Euler number and edge integrals. The former are divisible by $\left(t_{1}+t_{2}\right)$ by induction. We conclude the skewer integrals are divisible by $\left(t_{1}+t_{2}\right)$.

We now specialize to the localization analysis of the rubber integral,

$$
\left\langle\left[\mathcal{I}_{(d)}\right] \mid\left[\mathcal{I}_{(d-1, d)}\right]\right\rangle_{n}^{\sim}=\sum_{\Gamma \in G_{n}(\mu, v)}\left\langle\left[\mathcal{I}_{(d)}\right] \mid\left[\mathcal{I}_{(d-1,1)}\right]\right\rangle^{\Gamma \sim}
$$

Assume $\mu \neq v$. Since both skewer vertices and edges contribute factors of $\left(t_{1}+t_{2}\right)$,

$$
\left.\left\langle\left[\mathcal{I}_{(d)}\right] \mid\left[\mathcal{I}_{(d-1,1)}\right]\right\rangle_{n}^{\sim}=\left\langle\left[\mathcal{I}_{(d)}\right]\right|\left[\mathcal{I}_{(d-1,1)}\right]\right)^{\Gamma_{\mu, v}^{0} \sim} \bmod t_{1}+t_{2},
$$

where $\Gamma_{\mu, v}^{0}$ is the unique single edged chain with $S=\varnothing$. The Proposition 24 is then a consequence of Lemma 25. 


\subsection{Induction step: II}

By Lemma 23, Proposition 24, and the Hilbert scheme calculation of OkounkovPandharipande [31], we obtain

$$
\left\langle\left[\mathcal{I}_{(d)}\right]\left|-\sigma_{1}(F)\right|\left[\mathcal{I}_{(d-1,1)}\right]\right\rangle_{n}=\left\langle\mathrm{J}^{(d)}\left|\widetilde{\mathrm{M}}_{\sigma}-\widetilde{\mathrm{M}}_{\sigma}(0)\right| \mathrm{J}^{(d-1,1)}\right\rangle_{\mathcal{F}, n-d}
$$

completing the proof of Proposition 22.

\section{The level $(0,0)$ theory}

\subsection{The operator $M_{D}$}

Let $D$ denote the insertion of the relative condition $-\left(2,1^{d-2}\right)$ in the local DonaldsonThomas theory of curves. Let the operator $M_{D}$ on Fock space be defined by matrix elements

$$
\begin{aligned}
\left\langle\mu\left|\mathrm{M}_{D}\right| \nu\right\rangle_{\mathcal{F}} & =\mathrm{DT}(0 \mid 0,0)_{\mu, D, \nu} \\
& =-\mathrm{DT}(0 \mid 0,0)_{\mu,\left(2,1^{d-2}\right), \nu} .
\end{aligned}
$$

By definition, the insertion $D$ vanishes in degrees $d=0,1$.

Proposition $26 \mathrm{M}_{D}=\mathrm{M}-\frac{t_{1}+t_{2}}{2} \frac{(-q)+1}{(-q)-1}|\cdot|$.

Proof By applying the degeneration formula to the definition of $M_{\sigma}$, we obtain

$$
\begin{aligned}
\left\langle\mu\left|\mathrm{M}_{\sigma}\right| v\right\rangle_{\mathcal{F}} & =q^{-d}\left\langle\mu\left|-\sigma_{1}(F)\right| v\right\rangle \\
& =\sum_{\gamma} q^{-d} \mathrm{Z}(0 \mid 0,0)_{\mu, \gamma, \nu} \triangle_{d}(\gamma, \gamma) q^{-d}\left\langle\gamma \mid-\sigma_{1}(F)\right\rangle \\
& =\sum_{\gamma} \operatorname{DT}(0 \mid 0,0)_{\mu, \gamma, \nu} \triangle_{d}(\gamma, \gamma) q^{-d} \frac{\left\langle\gamma \mid-\sigma_{1}(F)\right\rangle}{\langle\varnothing \mid\rangle}
\end{aligned}
$$

By equation (18),

$$
\left\langle\gamma \mid-\sigma_{1}(F)\right\rangle=0
$$

if $\ell(\gamma)<d-1$. Hence, there are only two nonvanishing terms in the sum over the partition $\gamma$ :

$$
\begin{aligned}
\left\langle\mu\left|\mathrm{M}_{\sigma}\right| \nu\right\rangle_{\mathcal{F}}= & \operatorname{DT}(0 \mid 0,0)_{\mu,\left(1^{d}\right), \nu} \frac{d !\left(t_{1} t_{2}\right)^{d}}{q^{d}} \frac{\left\langle\left(1^{d}\right) \mid-\sigma_{1}(F)\right\rangle}{\langle\varnothing \mid\rangle} \\
& -\mathrm{DT}(0 \mid 0,0)_{\mu,\left(2,1^{d-2}\right), \nu} \frac{2(d-2) !\left(t_{1} t_{2}\right)^{d-1}}{q^{d}} \frac{\left\langle\left(2,1^{d-2}\right) \mid-\sigma_{1}(F)\right\rangle}{\langle\varnothing \mid\rangle}
\end{aligned}
$$


By Lemma 21 for the cap DT $(0 \mid 0,0)_{\lambda}$, the insertion $\left(1^{d}\right)$ can be freely added or removed in the local Donaldson-Thomas theory of curves. Hence,

$$
\begin{aligned}
\operatorname{DT}(0 \mid 0,0)_{\mu,\left(1^{d}\right), v} & =\operatorname{DT}(0 \mid 0,0)_{\mu, v} \\
& =\langle\mu \mid v\rangle_{\mathcal{F}},
\end{aligned}
$$

Similarly,

$$
\begin{aligned}
q^{-d} \frac{\left\langle\left(1^{d}\right) \mid-\sigma_{1}(F)\right\rangle}{\langle\varnothing \mid\rangle} & =q^{-d} \frac{\left\langle\left(1^{d}\right)\left|-\sigma_{1}(F)\right|\left(1^{d}\right)\right\rangle}{\langle\varnothing|\varnothing|} \\
& =\left\langle\left(1^{d}\right)\left|\mathrm{M}_{\sigma}\right|\left(1^{d}\right)\right\rangle \\
& =\frac{\left(t_{1}+t_{2}\right)}{d !\left(t_{1} t_{2}\right)^{d}}\left(\frac{d}{2} \frac{(-q)+1}{(-q)-1}-\Phi(q)\right),
\end{aligned}
$$

where the last equality is obtain from Proposition 22. Finally,

$$
\begin{aligned}
q^{-d} \frac{\left\langle\left(2,1^{d-2}\right) \mid-\sigma_{1}(F)\right\rangle}{\langle\varnothing \mid\rangle} & =q^{-d} \frac{\left\langle\left(2,1^{d-2}\right)\left|-\sigma_{1}(F)\right|\left(1^{d}\right)\right\rangle}{|\varnothing| \varnothing \mid} \\
& =\left\langle\left(2,1^{d-2}\right)\left|\mathrm{M}_{\sigma}\right|\left(1^{d}\right)\right\rangle_{\mathcal{F}} \\
& =-\left\langle\left(2,1^{d-2}\right) \mid\left(2,1^{d-2}\right)\right\rangle_{\mathcal{F}} .
\end{aligned}
$$

We conclude

$$
\begin{aligned}
\left\langle\mu\left|\mathrm{M}_{D}\right| v\right\rangle_{\mathcal{F}} & =-\mathrm{DT}(0 \mid 0,0)_{\mu,\left(2,1^{d-2}\right), v} \\
& =\left\langle\mu\left|\mathrm{M}_{\sigma}\right| v\right\rangle_{\mathcal{F}}-\left(t_{1}+t_{2}\right)\langle\mu \mid v\rangle_{\mathcal{F}}\left(\frac{d}{2} \frac{(-q)+1}{(-q)-1}-\Phi(q)\right) \\
& =\left\langle\mu\left|\mathrm{M}-\frac{t_{1}+t_{2}}{2} \frac{(-q)+1}{(-q)-1}\right| \cdot|| \nu\right\rangle_{\mathcal{F}},
\end{aligned}
$$

where the last equality is obtained from Proposition 22.

\subsection{Proof of Theorem 3 in level $(0,0)$}

9.2.1 Starred series The terminology of Bryan-Pandharipande [4] is convenient for discussing the GW/DT correspondence. On the Gromov-Witten side, let

$$
\mathrm{GW}^{*}\left(g \mid k_{1}, k_{2}\right)_{\lambda^{1} \ldots \lambda^{r}}=(-i u)^{d\left(2-2 g+k_{1}+k_{2}\right)-\delta} \mathrm{Z}^{\prime}(N)_{\lambda^{1} \ldots \lambda^{r}},
$$

where $N$ is rank 2 bundle of level $\left(k_{1}, k_{2}\right)$ on a genus $g$ curve and

$$
\delta=\sum_{i=1}^{r}\left(d-\ell\left(\lambda^{i}\right)\right) .
$$


On the Donaldson-Thomas side, let

$$
\begin{aligned}
\mathrm{DT}^{*}\left(g \mid k_{1}, k_{2}\right)_{\lambda^{1} \ldots \lambda^{r}} & =(-q)^{-\frac{d}{2}\left(2-2 g+k_{1}+k_{2}\right)} \mathrm{Z}_{D T}^{\prime}(N)_{\lambda^{1} \ldots \lambda^{r}} \\
& =(-1)^{-d(1-g)}(-q)^{-\frac{d}{2}\left(k_{1}+k_{2}\right)} \mathrm{DT}\left(g \mid k_{1}, k_{2}\right) .
\end{aligned}
$$

Theorem 3 of the Gromov-Witten/Donaldson-Thomas correspondence for local curves can be restated as the equality

$$
\mathrm{GW}^{*}\left(g \mid k_{1}, k_{2}\right)_{\lambda^{1} \ldots \lambda^{r}}=\mathrm{DT}^{*}\left(g \mid k_{1}, k_{2}\right)_{\lambda^{1} \ldots \lambda^{r}},
$$

after the variable change $e^{i u}=-q$,

9.2.2 TQFT Slightly altered metrics are defined for raising the indices of the starred series:

$$
\begin{aligned}
\mathrm{GW}^{*}\left(g \mid k_{1}, k_{2}\right)_{\mu^{1} \ldots \nu^{s}}^{v^{1} \ldots \mu^{t}} & =\left(\prod_{i=1}^{t} \mathfrak{z}\left(v^{i}\right)\left(-t_{1} t_{2}\right)^{l\left(v^{i}\right)}\right) \mathrm{GW}^{*}\left(g \mid k_{1}, k_{2}\right)_{\mu^{1} \ldots \mu^{s} v^{1} \ldots v^{t}}, \\
\mathrm{DT}^{*}\left(g \mid k_{1}, k_{2}\right)_{\mu^{1} \ldots \mu^{s}}^{v^{1} \ldots \nu^{t}} & =\left(\prod_{i=1}^{t} \mathfrak{z}\left(v^{i}\right)\left(-t_{1} t_{2}\right)^{l\left(v^{i}\right)}\right) \mathrm{DT}^{*}\left(g \mid k_{1}, k_{2}\right)_{\mu^{1} \ldots \mu^{s} v^{1} \ldots \nu^{t}}
\end{aligned}
$$

With respect to the above metrics, the starred partition function satisfy the same degeneration rules as their unstarred counterparts:

$$
\begin{aligned}
\mathrm{GW}^{*}\left(g \mid k_{1}, k_{2}\right)_{\mu^{1}, \ldots, \mu^{s}}^{v^{1}, \ldots, \nu^{t}} & =\sum_{\gamma} \mathrm{GW}^{*}\left(g^{\prime} \mid k_{1}^{\prime}, k_{2}^{\prime}\right)_{\mu^{1}, \ldots, \mu^{s}}^{\gamma} \mathrm{GW}^{*}\left(g^{\prime \prime} \mid k_{1}^{\prime \prime}, k_{2}^{\prime \prime}\right)_{\gamma}^{\nu^{1}, \ldots, \nu^{t}}, \\
\mathrm{DT}^{*}\left(g \mid k_{1}, k_{2}\right)_{\mu^{1}, \ldots, \mu^{s}}^{v^{1}, \ldots, \nu^{t}} & =\sum_{\gamma} \mathrm{DT}^{*}\left(g^{\prime} \mid k_{1}^{\prime}, k_{2}^{\prime}\right)_{\mu^{1}, \ldots, \mu^{s}}^{\gamma} \mathrm{DT}^{*}\left(g^{\prime \prime} \mid k_{1}^{\prime \prime}, k_{2}^{\prime \prime}\right)_{\gamma}^{v^{1}, \ldots, \nu^{t}},
\end{aligned}
$$

where $g=g^{\prime}+g^{\prime \prime}$, and $k_{i}=k_{i}^{\prime}+k_{i}^{\prime \prime}$, and

$$
\begin{aligned}
\mathrm{GW}^{*}\left(g \mid k_{1}, k_{2}\right)_{\mu^{1}, \ldots, \mu^{s}} & =\sum_{\gamma} \mathrm{GW}^{*}\left(g-1 \mid k_{1}, k_{2}\right)_{\mu^{1}, \ldots, \mu^{s}, \gamma}^{\gamma}, \\
\mathrm{DT}^{*}\left(g \mid k_{1}, k_{2}\right)_{\mu^{1}, \ldots, \mu^{s}} & =\sum_{\gamma} \mathrm{DT}^{*}\left(g-1 \mid k_{1}, k_{2}\right)_{\mu^{1}, \ldots, \mu^{s}, \gamma}^{\gamma} .
\end{aligned}
$$

Hence, tensor functors

$$
\mathbf{G W}^{*}, \mathbf{D T}^{*}: 2 \mathbf{C o b}^{L_{1}, L_{2}} \rightarrow \operatorname{Rmod} .
$$

can be defined just as before. 
9.2.3 Matching in level $(0,0)$ Using the TQFT structure, to prove Theorem 3 in level $(0,0)$, we must establish the following three equalities:

$$
\begin{aligned}
\mathrm{GW}^{*}(0 \mid 0,0)_{\lambda} & =\mathrm{DT}^{*}(0 \mid 0,0)_{\lambda}, \\
\mathrm{GW}^{*}(0 \mid 0,0)_{\lambda \mu} & =\mathrm{DT}^{*}(0 \mid 0,0)_{\lambda \mu}, \\
\mathrm{GW}^{*}(0 \mid 0,0)_{\lambda \mu \nu} & =\mathrm{DT}^{*}(0 \mid 0,0)_{\lambda \mu \nu},
\end{aligned}
$$

corresponding respectively to the cap, the tube, and the pair of pants.

The matching of the level $(0,0)$ cap is a consequence of [4, Lemma 6.2] for the Gromov-Witten side and Lemma 21 of Section 6 for the Donaldson-Thomas side. Similarly, the level $(0,0)$ tube matching is a consequence of [4, Lemma 6.1] and Lemma 4 of Section 3.

The pair of pants matching in level $(0,0)$ is more subtle. The main result of the Appendix of [4] is the unique determination of the level $(0,0)$ TQFT for the local Gromov-Witten theory of curves by the cap, the tube, and the set of series

$$
\mathrm{GW}^{*}(0 \mid 0,0)_{\mu,\left(2,1^{d-2}\right), \nu}
$$

for all $\mu$ and $\nu$. Since the cap and the tube have been shown to match, the equality

$$
\mathrm{GW}^{*}(0 \mid 0,0)_{\mu,\left(2,1^{d-2}\right), \nu}=\mathrm{DT}^{*}(0 \mid 0,0)_{\mu,\left(2,1^{d-2}\right), \nu}
$$

suffices to complete the matching in level $(0,0)$. Equality $(55)$ is a consequence of $[4$, A.3] and Proposition 26 above.

\section{The cap of level $(-1,0)$}

\subsection{TQFT}

By [4, Theorem 4.1], the proof of Theorem 3 for all levels now requires only the equality

$$
\mathrm{GW}^{*}(0 \mid-1,0)_{\lambda}=\mathrm{DT}^{*}(0 \mid-1,0)_{\lambda} \text {. }
$$

The Gromov-Witten side was calculated in Bryan-Pandharipande [3; 4]. By [4, Lemma 6.3 and Section 6.4.1],

$$
\mathrm{GW}^{*}(0 \mid-1,0)_{\lambda}=(-q)^{-d / 2} t_{2}^{-\ell(\lambda)} \frac{(-1)^{d-\ell(\lambda)}}{\mathfrak{z}(\lambda)} t_{2}^{-\ell(\lambda)} \prod_{i=1}^{\ell(\lambda)} \frac{1}{1-(-q)^{-\lambda_{i}}}
$$


In order to prove (56), we must find the following evaluation:

$$
\begin{aligned}
\operatorname{DT}(0 \mid-1,0)_{\lambda} & =(-1)^{d}(-q)^{-d / 2} \mathrm{DT}^{*}(0 \mid-1,0)_{\lambda} \\
& =t_{2}^{-\ell(\lambda)} \frac{(-1)^{d-\ell(\lambda)}}{\mathfrak{z}(\lambda)} q^{-d} \prod_{i=1}^{\ell(\lambda)} \frac{1}{1-(-q)^{-\lambda_{i}}} \\
& =\frac{t_{2}^{-\ell(\lambda)}}{\mathfrak{z}(\lambda)} \prod_{i=1}^{\ell(\lambda)} \frac{1}{1-(-q)^{\lambda_{i}}} .
\end{aligned}
$$

\subsection{T-action}

Let $T$ be the standard 2-dimensional torus action on the bundle

$$
N=\mathcal{O}(-1) \oplus \mathcal{O}
$$

over $\mathbf{P}^{1}$ with scaling weights $t_{1}$ and $t_{2}$ on the factors $\mathcal{O}(-1)$ and $\mathcal{O}$ respectively. Let the 1-dimensional torus $S$ act with weights $s,-s$ at the fixed points

$$
0, \infty \in \mathbf{P}^{1}
$$

and weights $(-s, 0)$ on the fiber of $N_{\infty}$. We will consider the full

$$
\mathbf{T}=S \times T
$$

action on $N$.

\subsection{Independence}

Let $L \subset N$ denote the $\mathbf{T}$-equivariant divisor determined by the summand $\mathcal{O}(-1)$, and let

$$
[L] \in A_{\mathbf{T}}^{1}(N, \mathbb{Q})
$$

be the associated class. Let

$$
\lambda([L])=\left\{\lambda_{1}([L]), \ldots, \lambda_{\ell(\lambda)}([L])\right\}
$$

be a weighted partition of $d$. The reduced $\mathbf{T}$-equivariant Donaldson-Thomas residue integral relative to $N_{\infty}$,

$$
\langle\mid \lambda([L])\rangle_{(-1,0)}^{\prime}=\frac{\left\langle|\lambda([L])|_{d}^{(-1,0)}\right.}{\left\langle|\varnothing|_{0}^{(-1,0)}\right.},
$$

is of degree 0 in the equivariant parameters $s, t_{1}$, and $t_{2}$. 
Lemma $27\langle\mid \lambda([L])\rangle_{(-1,0)}^{\prime}$ is independent of $s, t_{1}$, and $t_{2}$.

Proof Let $N \subset \bar{N}$ be a $\mathbf{T}$-equivariant compactification, and let

$$
[P] \in H_{2}(\bar{N}, \mathbb{Z})
$$

be the push-forward to $\bar{N}$ of the class of the zero section $P \subset N$. Consider the restricted moduli space of ideal sheaves

$$
R=\epsilon_{\infty}^{-1}\left(C_{\lambda[L]}\right) \subset I_{n}\left(\bar{N} / \bar{N}_{\infty}, d[P]\right),
$$

following the notation of Section 1.2.3. Since the line bundle $\mathcal{O}(-1)$ over $\mathbf{P}^{1}$ has no nontrivial multisections, the elements of

$$
\left[I_{Z}\right] \in R_{n}
$$

for which the entire 1-dimension support of $Z$ lies on $P$ determine an open and closed T-equivariant substack $R_{n}^{0} \subset R_{n}$

Dimension 0 integrals over $R_{n}^{0}$ are certainly independent of the equivariant parameters $s, t_{1}$, and $t_{2}$. By localization,

$$
\langle\mid \lambda([L])\rangle_{(-1,0)}^{\prime}=\frac{\sum_{n} q^{n} \int_{\left[R_{n}^{0}\right]^{\mathrm{vir}} 1}}{\sum_{n} q^{n} \int_{\left[I_{n}\left(\bar{N} / \bar{N}_{\infty}, 0\right)\right]^{\mathrm{vir}}} 1} .
$$

Since the denominator on the right is also independent of the equivariant parameters, the Lemma is proven.

\subsection{Localization}

The $\mathbf{T}$-equivariant virtual localization formula for the series

$$
\langle\mid \lambda([L])\rangle_{(-1,0)}^{\prime}
$$

involves an edge summation over all of the $T$-fixed points $\mathcal{I}_{\mu}$ of the Hilbert scheme $\operatorname{Hilb}\left(N_{\infty}, d\right)$. See Maulik et al $[24 ; 25]$ for a discussion of localization in relative Donaldson-Thomas theory.

We orient the partition $\mu$ so that the rows of the associated Young diagram extend in the $\mathcal{O}$ direction. Define $n(\mu)$ by a summation over rows:

$$
n(\mu)=\sum_{i=1}^{\ell(\mu)}(i-1) \mu_{i}
$$

With our orientation conventions, $d+n(\mu)$ is the Euler characteristic of a pure edge with profile $\mu$. 
By an application of the virtual localization formula, we find the series (57) equals

$$
\sum_{|\mu|=d} \frac{\left.\mathrm{W}(\mu, \varnothing, \varnothing)\right|_{s, t_{1}-s, t_{2}}}{\left.\mathrm{~W}(\varnothing, \varnothing, \varnothing)\right|_{s, t_{1}-s, t_{2}}} \cdot q^{n(\mu)} \mathrm{E}^{(-1,0)}(\mu) \cdot \frac{\left\langle\left[\mathcal{I}_{\mu}\right]\left|\frac{1}{-s-\psi_{\infty}}\right| \lambda\right\rangle^{\sim}}{\left\langle\left.\varnothing\left|\frac{1}{-s-\psi_{\infty}}\right| \varnothing\right|^{\sim}\right.} t_{2}^{\ell(\lambda)} .
$$

The terms $W$ and $E^{(-1,0)}$ are respectively the equivariant vertex and the equivariant edge weight $[24 ; 25]$. The rubber integral series in the numerator is

$$
\left\langle\left[\mathcal{I}_{\mu}\right]\left|\frac{1}{-s-\psi_{\infty}}\right| \lambda\right\rangle^{\sim}=q^{d}\left\langle\left[\mathcal{I}_{\mu}\right] \mid \lambda\right\rangle_{d, d}+\sum_{n>d} q^{n}\left\langle\left[\mathcal{I}_{\mu}\right]\left|\frac{1}{-s-\psi_{\infty}}\right| \lambda\right\rangle_{n, d}^{\sim} .
$$

The denominator series has a parallel definition.

By Lemma 27, the series (57) is independent $s, t_{1}$, and $t_{2}$. Hence the localization formula can be evaluated after specialization of the equivariant parameters.

\subsection{Rubber}

We evaluate the localization formula (57) after the specialization

$$
t_{1}+t_{2}=0 \text {. }
$$

By the vanishing of Lemma 13, the rubber integrals on the right side simplify to

$$
\frac{\left\langle\left[\mathcal{I}_{\mu}\right]\left|\frac{1}{-s-\psi_{\infty}}\right| \lambda\right\rangle^{\sim}}{\left\langle\varnothing\left|\frac{1}{-s-\psi_{\infty}}\right| \varnothing\right\rangle^{\sim}}=q^{d}\left\langle\left[\mathcal{I}_{\mu}\right] \mid \lambda\right\rangle_{d, d} .
$$

The matrix element on the right is the equivariant intersection form of the classical cohomology of $\operatorname{Hilb}\left(N_{\infty}, d\right)$.

For opposite weights (60), the Hilbert scheme intersections reduce to characters of the symmetric group, see for example Vasserot [35]. We find

$$
\left\langle\left[\mathcal{I}_{\mu}\right] \mid \lambda\right\rangle_{d, d}=\frac{t_{2}^{d-\ell(\lambda)}}{\mathfrak{z}(\lambda)} \chi_{\lambda}^{\mu} \prod_{\square \in \mu} h(\square) .
$$

Here, $h(\square)$ denotes the hook length. A similar calculation can be found in OkounkovPandharipande [31].

\subsection{Edge}

The edge term of the localization formula (59) also simplifies after the specialization (57). 
We recall the formula for the edge term adapted to the level $(-1,0)$ geometry (see Maulik et al [24]). Given a partition $\mu$, form the following polynomials

$$
\begin{aligned}
Q_{\mu}\left(x_{1}, x_{2}\right) & =\sum_{(i, j) \in \mu} x_{1}^{i} x_{2}^{j}, \\
F_{\mu}\left(x_{1}, x_{2}\right) & =-Q_{\mu}-\frac{\bar{Q}_{\mu}}{x_{1} x_{2}}+Q_{\mu} \bar{Q}_{\mu} \frac{\left(1-x_{1}\right)\left(1-x_{2}\right)}{x_{1} x_{2}} .
\end{aligned}
$$

The sum in the first definition is over the interior corners of the squares of the Young diagram of $\mu-$ the corners closest to the origin. Also,

$$
\bar{Q}_{\mu}\left(x_{1}, x_{2}\right)=Q_{\mu}\left(x_{1}^{-1}, x_{2}^{-1}\right) .
$$

The rational function

$$
E_{\mu}=\frac{F_{\mu}\left(x_{1}, x_{2}\right)}{x_{0}-1}+\frac{F_{\mu}\left(x_{1} x_{0}, x_{2}\right)}{x_{0}^{-1}-1}
$$

is readily seen to be a Laurent polynomial in the variables $x_{i}$. The edge weight $\mathrm{E}(\mu)$ is obtained from the following transformation:

$$
E_{\mu}=\sum_{k} a_{k} x_{0}^{k_{0}} x_{1}^{k_{1}} x_{2}^{k_{2}} \mapsto \mathrm{E}(\mu)=\prod_{k}\left(k_{0} s+k_{1}\left(t_{1}-s\right)+k_{2} t_{2}\right)^{-a_{k}} .
$$

Setting $t_{1}+t_{2}=0$ is equivalent to substituting

$$
x_{0}=\left(x_{1} x_{2}\right)^{-1}
$$

in the above formulas.

Lemma 28 We have

$$
F_{\mu}\left(x_{1}, x_{2}\right)=-\sum_{\square \in \mu}\left(x_{1}^{l(\square)} x_{2}^{-a(\square)-1}+x_{1}^{-l(\square)-1} x_{2}^{a(\square)}\right),
$$

where $a(\square)$ and $l(\square)$ denote the arm-lengths and leg-length of a square in a diagram (number of squares to the right and below $\square$, respectively).

Proof The polynomial $F_{\mu}$ is, up to sign, the character of the scaling torus action on the tangent space at $\left[\mathcal{I}_{\mu}\right]$ to the Hilbert scheme of points in $\mathbb{C}^{2}$. The exponents in the formula are well known to be the weights of tangent action, see for example Haiman [11].

Define an auxiliary function

$$
G\left(x_{1}, x_{2}\right)=-\sum_{\square \in \mu} x_{1}^{l(\square)} x_{2}^{-a(\square)-1}
$$


for which $F=G+\bar{G} /\left(x_{1} x_{2}\right)$. We compute

$$
\left.E_{\mu}\right|_{x_{3}=\left(x_{1} x_{2}\right)^{-1}}=-\left.G\right|_{x_{1}=x_{2}^{-1}}+\frac{G-\left.G\right|_{x_{1}=x_{2}^{-1}}}{\left(x_{1} x_{2}\right)^{-1}-1}-\frac{\bar{G}-\left.\bar{G}\right|_{x_{2}=x_{1}^{-1}}}{\left(x_{1} x_{2}\right)-1} .
$$

All three terms of (63) are Laurent polynomials. The third term is minus bar of the second one. By the transformation (62), the factors corresponding to the second and third terms of (63) cancel up to a sign. This sign is the parity of the overall number of monomials in the second term in (63) equal to

$$
\sum_{\square \in \mu} l(\mu)=n(\mu)
$$

We have proven the following result:

$$
\left.\mathrm{E}(\mu)^{(-1,0)}\right|_{s, t_{1}-s, t_{2}}=(-1)^{n(\mu)} t_{2}^{-d} \prod_{\square \in \mu} h(\square)^{-1} .
$$

\subsection{Vertex}

The $\mathbf{T}$-equivariant tangent weights of $N$ at the fixed point over $0 \in \mathbf{P}^{1}$ satisfy the Calabi-Yau condition after the specialization (60):

$$
s+\left(t_{1}-s\right)+t_{2}=0 .
$$

The vertex $\mathrm{W}(\mu, \varnothing, \varnothing)$ has a rather simple evaluation in the Calabi-Yau case (see Maulik et al [24] and Okounkov-Reshetikhin-Vafa [33]):

$$
\left.\mathrm{W}(\mu, \varnothing, \varnothing)\right|_{s, t_{1}-s, t_{2}}=\sum_{\pi}(-q)^{|\pi|} .
$$

The sum is over all 3-dimensional partitions $\pi$ with a single infinite leg in the $s$ direction asymptotic to $\mu$. Here, $|\pi|$ is the renormalized volume - the number of boxes remaining after the infinite leg is removed. In particular,

$$
\left.\mathrm{W}(\varnothing, \varnothing, \varnothing)\right|_{s, t_{1}-s, t_{2}}=M(-q),
$$

a specialization of (6).

Evaluation of the Calabi-Yau vertex is reduced to the enumeration of 3-dimensional partition. The enumeration for the 1-legged vertex is solved in Okounkov-Reshetikhin [32],

$$
\frac{\left.\mathrm{W}(\mu, \varnothing, \varnothing)\right|_{s, t_{1}-s, t_{2}}}{\left.\mathrm{~W}(\varnothing, \varnothing, \varnothing)\right|_{s, t_{1}-s, t_{2}}}=\prod_{\square \in \mu} \frac{1}{1-(-q)^{h(\square)}}
$$


where the product is over all squares in the Young diagram of $\mu$.

The origin of hook lengths in (64) is the following classical formula (see Macdonald [22]) for the value of the Schur function $s_{\mu}$ at the point $\left(1, q, q^{2}, \ldots\right)$,

$$
s_{\mu}\left(1, q, q^{2}, \ldots\right)=q^{n(\mu)} \prod_{\square \in \mu} \frac{1}{1-q^{h(\square)}} .
$$

\subsection{Evaluation}

Putting all pieces of the localization formula (59) together, we find:

$$
\left\langle\lambda([L])||_{(0,-1)}^{\prime}=\frac{q^{d}}{\mathfrak{z}(\lambda)} \sum_{\mu} \chi_{\lambda}^{\mu} s_{\mu}\left(1,-q,(-q)^{2},(-q)^{3}, \ldots\right) .\right.
$$

By a classical formula in the theory of symmetric functions, $\sum_{\mu} \chi_{\lambda}^{\mu} s_{\mu}$ equals the power sum symmetric function $p_{\lambda}$, see [22]. Since

$$
p_{k}\left(1,-q,(-q)^{2},(-q)^{3}, \ldots\right)=\frac{1}{1-(-q)^{k}},
$$

we obtain the following result.

\section{Proposition 29}

$$
\langle\lambda([L])| Y_{(0,-1)}^{\prime}=\frac{q^{d}}{\mathfrak{z}(\mu)} \prod_{i=1}^{\ell(\lambda)} \frac{1}{1-(-q)^{\lambda_{i}}}
$$

\subsection{Proof of Theorem 3}

By definition, we find

$$
\langle\mu([L]) \mid\rangle_{(0,-1)}^{\prime}=t_{2}^{\ell(\lambda)} q^{d} \mathrm{DT}(0 \mid-1,0) .
$$

Hence, by Proposition 29,

$$
\operatorname{DT}(0 \mid-1,0)=\frac{t_{2}^{-\ell(\lambda)}}{\mathfrak{z}(\mu)} \prod_{i=1}^{\ell(\lambda)} \frac{1}{1-(-q)^{\lambda_{i}}} .
$$

The matching $(56)$ of the $(-1,0)$ cap is established, and the proof of Theorem 3 is complete. 


\subsection{Proof of Theorem 2}

By Theorem 3, the rationality of the Donaldson-Thomas series for local curves is a direct consequence of Theorem 6.4 of Bryan-Pandharipande [4]. The proof of Theorem 2 is complete.

\section{The 1-legged vertex}

\subsection{Overview}

The localization formula for the level $(-1,0)$ cap together with a differential equation for rubber integrals provides an effective determination of the 1-legged equivariant vertex.

\subsection{Differential equation}

Consider the following Donaldson-Thomas rubber descendent series:

$$
\left\langle\mu\left|\frac{1}{1-\psi_{\infty}}\right| v\right\rangle^{\sim}=q^{d}\langle\mu \mid v\rangle_{d, d}+\sum_{n>d} q^{n}\left\langle\mu\left|\frac{1}{1-\psi_{\infty}}\right| v\right\rangle_{n, d}^{\sim} .
$$

Define a operator $\mathrm{S}$ on Fock space by the matrix elements

$$
\langle\mu|\mathrm{S}| v\rangle_{\mathcal{F}}=q^{-d} M(-q)^{t_{1}+t_{2}}\left\langle\mu\left|\frac{1}{1-\psi_{\infty}}\right| v\right\rangle^{\sim} \text {. }
$$

By the rubber calculus relation (29),

$$
q \frac{d}{d q} q^{-d}\left\langle\mu\left|\frac{1}{1-\psi_{\infty}}\right| \nu\right\rangle^{\sim}=q^{-d}\left\langle\mu\left|\frac{-\sigma_{1}(F)}{1-\psi_{\infty}}\right| \nu\right\rangle-q^{-d}\left\langle\mu\left|\frac{1}{1-\psi_{\infty}}\right| D \cdot \nu\right\rangle^{\sim} .
$$

By the topological recursion relation of Section 4.8 ,

$$
q^{-d}\left\langle\mu\left|\frac{-\sigma_{1}(F)}{1-\psi_{\infty}}\right| v\right\rangle=q^{-2 d} \sum_{\eta}\left\langle\mu\left|-\sigma_{1}(F)\right| \eta\right\rangle \triangle_{d}(\eta, \eta)\left\langle\eta\left|\frac{1}{1-\psi_{\infty}}\right| \nu\right\rangle^{\sim}
$$

Together with Proposition 22, we conclude

$$
q \frac{d}{d q} \mathrm{~S}=\mathrm{MS}-\mathrm{SM}(0)
$$

where $\mathrm{M}(0)$ denotes the $q$-constant terms of $\mathrm{M}$. The series $\Phi(d)$ drops out of right side of (68).

The differential equation (68) for rubber descendents is almost identical to the quantum differential equation for the Hilbert scheme of points of the plane (see OkounkovPandharipande [31]). 
Lemma $30\left\langle\left.\varnothing\left|\frac{1}{1-\psi_{\infty}}\right| \varnothing\right|^{\sim}=M(-q)^{-\left(t_{1}+t_{2}\right)}\right.$

Proof The differential equation (68) takes a simple form in degree 0:

$$
q \frac{d}{d q}\langle\varnothing|\mathrm{S}| \varnothing\rangle_{\mathcal{F}}=0 .
$$

The solution is a constant. The Lemma follows from definition (67).

By Lemma 30, we may express the matrix elements of $S$ as ratios of Donaldson-Thomas rubber series:

$$
\langle\mu|\mathrm{S}| \nu\rangle_{\mathcal{F}}=q^{-d} \frac{\left\langle\mu\left|\frac{1}{1-\psi_{\infty}}\right| \nu\right\rangle^{\sim}}{\left\langle\varnothing\left|\frac{1}{1-\psi_{\infty}}\right| \varnothing\right\rangle^{\sim}}
$$

\subsection{Computation of the 1-legged vertex}

Let $\mathrm{W}^{\prime}(\mu, \varnothing, \varnothing)$ denote the reduced 1-legged vertex,

$$
\mathrm{W}^{\prime}(\mu, \varnothing, \varnothing)=\frac{\mathrm{W}(\mu, \varnothing, \varnothing)}{\mathrm{W}(\mu, \varnothing, \varnothing)} .
$$

The localization formula (59),

$\langle\mid \lambda([L])\rangle_{(-1,0)}^{\prime}=$

$$
\left.\sum_{|\mu|=d} \mathrm{~W}^{\prime}(\mu, \varnothing, \varnothing)\right|_{s, t_{1}-s, t_{2}} \cdot q^{n(\mu)} \mathrm{E}^{(-1,0)}(\mu) \cdot \frac{\left\langle\left[\mathcal{I}_{\mu}\right]\left|\frac{1}{-s-\psi_{\infty}}\right| \lambda\right)^{\sim}}{\left\langle\left.\varnothing\left|\frac{1}{-s-\psi_{\infty}}\right| \varnothing\right|^{\sim}\right.} t_{2}^{\ell(\lambda)},
$$

has a fixed evaluation given by Proposition 29 independent of the equivariant parameters. If the matrix

$$
\mathrm{E}^{(-1,0)}(\mu) \cdot \frac{\left\langle\left[\mathcal{I}_{\mu}\right]\left|\frac{1}{-s-\psi_{\infty}}\right| \lambda\right\rangle^{\sim}}{\left\langle\left.\varnothing\left|\frac{1}{-s-\psi_{\infty}}\right| \varnothing\right|^{\sim}\right.}
$$

can be calculated, the above localization formula may be viewed as a square system of linear equations for the unknown vector $\mathrm{W}^{\prime}(\mu, \varnothing, \varnothing)$, where $\mu$ ranges over all partitions of a fixed size.

The matrix (70) is a product of two factors. The first is an invertible diagonal matrix of edge weights explicitly determined by (62). The second is the operator $\mathrm{S}$ written on 
the left in the fixed point basis. The operator $\mathrm{S}$ is completely determined by the linear differential equation (68). The inverse of $S$ satisfies

$$
q \frac{d}{d q} \mathrm{~S}^{-1}=\mathrm{M}(0) \mathrm{S}^{-1}-\mathrm{S}^{-1} \mathrm{M}
$$

In particular, $\mathrm{S}$ is invertible for $q$ not equal to a root of unity.

An identical argument can be used in the local Gromov-Witten theory of curves to determine 1-partition Hodge integrals from the results of Bryan-Pandharipande [4]. For Calabi-Yau Hodge integrals, the results specialize to the Gopakumar-Mariño-Vafa formula proven in Liu-Liu-Zhou [21] and Okounkov-Pandharipande [29].

Alternatively, both the 1-legged vertex and the 1-partition Hodge integrals can be recovered from an parallel localization formula for the level $(0,0)$ cap. We leave the details to the reader.

\section{References}

[1] R Bott, A residue formula for holomorphic vector-fields, J. Differential Geometry 1 (1967) 311-330 MR0232405

[2] J Bryan, T Graber, The crepant resolution conjecture, from: "Algebraic geometry Seattle 2005. Part 1", Proc. Sympos. Pure Math. 80, Amer. Math. Soc., Providence, RI (2009) 23-42 MR2483931

[3] J Bryan, R Pandharipande, Curves in Calabi-Yau threefolds and topological quantum field theory, Duke Math. J. 126 (2005) 369-396 MR2115262

[4] J Bryan, R Pandharipande, The local Gromov-Witten theory of curves, J. Amer. Math. Soc. 21 (2008) 101-136 MR2350052With an appendix by Bryan, C. Faber, A. Okounkov and Pandharipande

[5] S K Donaldson, R P Thomas, Gauge theory in higher dimensions, from: "The geometric universe (Oxford, 1996)", Oxford Univ. Press, Oxford (1998) 31-47 MR1634503

[6] Y Eliashberg, A Givental, H Hofer, Introduction to symplectic field theory, Geom. Funct. Anal. (2000) 560-673 MR1826267GAFA 2000 (Tel Aviv, 1999)

[7] A Gholampour, On the equivariant Gromov-Witten theory of $\mathbb{P}^{2}$-bundles over curves, Comm. Anal. Geom. 14 (2006) 633-671 MR2273289

[8] A Gholampour, Y Song, Evidence for the Gromov-Witten/Donaldson-Thomas correspondence, Math. Res. Lett. 13 (2006) 623-630 MR2250496

[9] T Graber, R Pandharipande, Localization of virtual classes, Invent. Math. 135 (1999) 487-518 MR1666787 
[10] I Grojnowski, Instantons and affine algebras I: The Hilbert scheme and vertex operators, Math. Res. Lett. 3 (1996) 275-291 MR1386846

[11] M Haiman, Notes on Macdonald polynomials and the geometry of Hilbert schemes, from: "Symmetric functions 2001: surveys of developments and perspectives", NATO Sci. Ser. II Math. Phys. Chem. 74, Kluwer Acad. Publ., Dordrecht (2002) 1-64 MR2059359

[12] E-N Ionel, T H Parker, Relative Gromov-Witten invariants, Ann. of Math. (2) 157 (2003) 45-96 MR1954264

[13] Y-H Kiem, J Li, Gromov-Witten invariants of varieties with holomorphic 2-forms arXiv:0707.2986

[14] M Lehn, Chern classes of tautological sheaves on Hilbert schemes of points on surfaces, Invent. Math. 136 (1999) 157-207 MR1681097

[15] M Lehn, C Sorger, Symmetric groups and the cup product on the cohomology of Hilbert schemes, Duke Math. J. 110 (2001) 345-357 MR1865244

[16] A-M Li, Y Ruan, Symplectic surgery and Gromov-Witten invariants of Calabi-Yau 3-folds, Invent. Math. 145 (2001) 151-218 MR1839289

[17] J Li, A degeneration formula of GW-invariants, J. Differential Geom. 60 (2002) 199293 MR1938113

[18] J Li, private communication (2004)

[19] J Li, B Wu, Degeneration of Donaldson-Thomas invariants, preprint (2009)

[20] W-p Li, Z Qin, W Wang, Vertex algebras and the cohomology ring structure of Hilbert schemes of points on surfaces, Math. Ann. 324 (2002) 105-133 MR1931760

[21] C-C Liu, K Liu, J Zhou, A proof of a conjecture of Mariño-Vafa on Hodge integrals arXiv:math.AG/0308015

[22] I G Macdonald, Symmetric functions and Hall polynomials, second edition, Oxford Mathematical Monographs, The Clarendon Press Oxford University Press, New York (1995) MR1354144 With contributions by A Zelevinsky, Oxford Science Publications

[23] M Mariño, C Vafa, Framed knots at large $N$ arXiv:hep-th/0108064

[24] D Maulik, N Nekrasov, A Okounkov, R Pandharipande, Gromov-Witten theory and Donaldson-Thomas theory I, Compos. Math. 142 (2006) 1263-1285 MR2264664

[25] D Maulik, N Nekrasov, A Okounkov, R Pandharipande, Gromov-Witten theory and Donaldson-Thomas theory II, Compos. Math. 142 (2006) 1286-1304 MR2264665

[26] D Maulik, R Pandharipande, A topological view of Gromov-Witten theory, Topology 45 (2006) 887-918 MR2248516

[27] D Maulik, R Pandharipande, $\mathbf{R}$ Thomas, Curves on $K 3$ surfaces and modular forms arXiv: 1001.2719 
[28] H Nakajima, Lectures on Hilbert schemes of points on surfaces, University Lecture Series 18, American Mathematical Society, Providence, RI (1999) MR1711344

[29] A Okounkov, R Pandharipande, Hodge integrals and invariants of the unknot, Geom. Topol. 8 (2004) 675-699 MR2057777

[30] A Okounkov, R Pandharipande, Virasoro constraints for target curves, Invent. Math. 163 (2006) 47-108 MR2208418

[31] A Okounkov, R Pandharipande, Quantum cohomology of the Hilbert scheme of points in the plane, Invent. Math. 179 (2010) 523-557 MR2587340

[32] A Okounkov, N Reshetikhin, Correlation function of Schur process with application to local geometry of a random 3-dimensional Young diagram, J. Amer. Math. Soc. 16 (2003) 581-603 MR1969205

[33] A Okounkov, N Reshetikhin, C Vafa, Quantum Calabi-Yau and classical crystals arXiv:hep-th/0310061

[34] R P Thomas, A holomorphic Casson invariant for Calabi-Yau 3-folds, and bundles on K3 fibrations, J. Differential Geom. 54 (2000) 367-438 MR1818182

[35] E Vasserot, Sur l'anneau de cohomologie du schéma de Hilbert de $\mathbb{C}^{2}$, C. R. Acad. Sci. Paris Sér. I Math. 332 (2001) 7-12 MR1805619

Department of Mathematics, Princeton University

Princeton NJ 08544, USA

okounkov@math.princeton.edu, rahulp@math.princeton.edu

Proposed: Jim Bryan

Seconded: Lothar Goettsche, Richard Thomas
Received: 22 January 2010

Accepted: 2 May 2010 